\title{
AN ACCURATE FIC-FEM FORMULATION FOR THE 1D CONVECTION-DIFFUSION-REACTION EQUATION
}

\author{
E. Oñate ${ }^{1,2}$, J. Miquel ${ }^{1,2}$ and P. Nadukandi ${ }^{1}$ \\ 1 Centre Internacional de Mètodes Numèrics en Enginyeria (CIMNE) \\ ${ }^{2}$ Universitat Politècnica de Catalunya \\ Campus Norte UPC, 08034 Barcelona, Spain
}

\begin{abstract}
In this paper we present an accurate stabilized FIC-FEM formulation for the 1D advection-diffusion-reaction equation in the exponential and propagation regimes using two stabilization parameters. Both the steady-state and transient solutions are considered.

The stabilized formulation is based on the standard Galerkin FEM solution of the governing differential equations derived via the Finite Increment Calculus (FIC) method. The steady-state problem is considered first. The optimal value of the two stabilization parameters ensuring an exact (nodal) FEM solution using uniform meshes of linear 2-noded elements is obtained. In the absence of the absorption term the formulation simplifies to the standard one-parameter Petrov-Galerkin method for the advection-diffusion problem. For the diffusion-reaction case one stabilization parameter is just needed and the diffusion-type stabilization term is identical to that obtained by Felippa and Oñate [16] using a variational FIC approach. A procedure for computing the stabilization parameters for the transient problem is proposed. The accuracy of the new FIC-FEM formulation is demonstrated in the solution of steady-state and transient 1D advection-diffusion-radiation problems for a the range of physical parameters and boundary conditions. Finally we outline the procedure to extend the 1D FIC-FEM formulation to multidimensions.
\end{abstract}

Key words: Finite element method, stabilized formulation, convection-diffusion-reaction, finite increment calculus, finite calculus, FIC, one dimensional problem

\section{INTRODUCTION}

Considerable effort has been spent in recent years for deriving finite element methods (FEM) for the solution of the advection-diffusion-reaction equation. The physical behaviour of this equation is varied in terms of the values of the diffusion coefficient. In the propagation regime, originated by large values of the productive term versus the diffusion one (the Helmholtz equation), solutions are exponentially modulated sinusoidal functions. Typical problems found here in the numerical solution are those of phase, amplitude and pollution errors. In the exponential regime, solutions are of the form of real exponential functions where absorptive (dissipative) or productive source terms are possible. Numerical schemes here find difficulties to approximate the sharp gradients appearing in the neighborhood of boundary and internal layers in two (2D) and three (3D) dimensions due to high Peclet and/or Damköhler numbers.

It is well known that the numerical solution of this problem by the Bubnov-Galerkin FEM is prone to exhibit global, Gibbs and dispersive oscillations. The solution of the stationary problem by the above method exhibits spurious global oscillations for the convection-dominated case. The local Gibbs oscillations are exhibited along the characteristic layers for the 2D/3D convectiondominated case. For the reaction-dominated cases Gibbs oscillations may be found near the Dirichlet boundaries and in the regions where the distributed source term is nonregular. The solution of the transient problem may exhibit dispersive oscillations when the initial solution and/or the distributed source term are nonregular. 
In the context of variational formulations and weighted residual methods, control over the global instability has been achieved via the streamline-upwind Petrov-Galerkin (SUPG) [6, 27], Taylor-Galerkin [13], characteristic Galerkin [15, 37], Galerkin least squares (GLS) [29], bubble functions [1, 3, 4], variational multiscale (VMS) [30], characteristic-based split (CBS) [67] and finite increment calculus (also known as finite calculus) (FIC) based methods [42, 44, 45, 53, 54, 57]. A thorough comparison of some of these methods can be found in [9]. Oñate and Manzan [44, 45] showed that many of the above stabilized methods can be recovered using the FIC equations via an appropriate definition of the stabilization parameters. Nevertheless nonregular solutions continue to exhibit the Gibbs and dispersive oscillations.

Several shock-capturing nonlinear Petrov-Galerkin methods were proposed to control the Gibbs oscillations observed across characteristic internal/boundary layers for the convection-diffusion problem $[8,11,16,22,34,35,38,53]$. A thorough review, comparison and state of the art of these and several other shock-capturing methods for the convection-diffusion equations was done in [33]. Reactive terms were not considered in the design of these methods and hence they fail to control the localized oscillations in the presence of these terms. Exceptions to this are the consistent approximate upwind (CAU) method [22], the methods presented in [7] and those that take the CAU method as the starting point [16]. Nevertheless, the expressions for the stabilization parameters therein were never optimized for reactive instability and often the solutions are over-diffusive in these cases.

In the quest to gain reactive stability several methods were built upon the existing frameworks of methods that control global oscillations. Following the framework of the SUPG method linear Petrov-Galerkin methods were proposed for the convection-diffusion-reaction problem, viz. the DRD $[65]$ and $(S U+C) P G[31]$ methods. Based on the GLS method, linear stabilized methods were

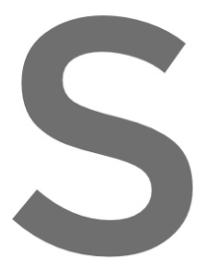
proposed, viz. the GGLS method [18] for the diffusion-reaction problem and the GLSGLS method [23] for the convection-diffusion-production probl
bubbles the USFEM method [19] for the diffusion-1
[20] and the link cutting bubbles procedure [5] for
proposed. Based on the VMS method linear stabil
diffusion-reaction problem, viz. the ASGS meth
the SGS-GSGS method [26]. Using the FIC eq
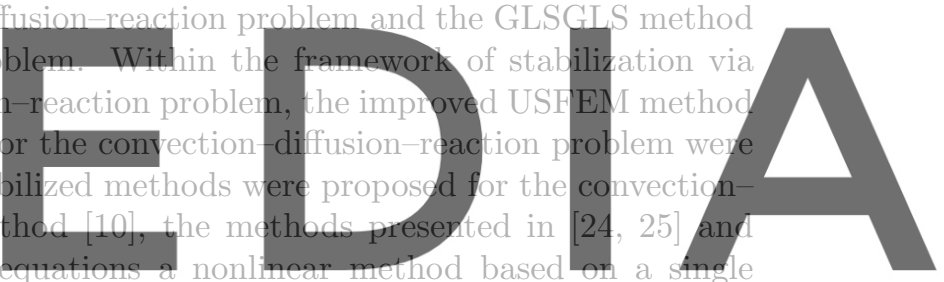

stabilization parameter was proposed for the convection-diffusion-absorption problem in 1D [54]

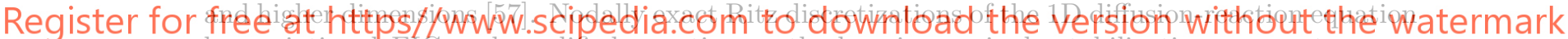
by variational FIC and modified equation methods using a single stabilization parameter were presented in [17]

In [39] we presented the design of a FIC-based nonlinear high-resolution Petrov-Galerkin (HRPG) method for the 1D convection-diffusion-reaction problem. The method is capable of reproducing high-resolution numerical solutions for both the stationary (efficient control of global and Gibbs oscillations and transient regimes (efficient control of dispersive oscillations. In [40] we presented the statement of the problem and the HRPG method in higher-dimensions.

Generally the homogeneous steady convection-diffusion-reaction problem in 1D has two fundamental solutions. Likewise, the characteristic equation associated with linear stabilized methods which result in compact stencils are quadratic and hence have two solutions. Thus in principle using two stabilization parameters (independent of the boundary conditions) linear stabilized methods which result in compact stencils can be designed to be nodally exact in 1D. Following this line several "two-parameter methods" were designed to be nodally exact for the stationary problem in $1 \mathrm{D}[5,23,26]$.

Control over the dispersive oscillations for the transient convection-diffusion problem via linear Petrov-Galerkin methods were discussed in [32] and using space-time finite elements in [66]. As for the linear methods, optimizing the expressions of the stabilization parameters to attain monotonicity will lead to solutions that are at most first-order accurate.

In this paper we present an accurate FIC-FEM formulation for the 1D advection-diffusionreaction equation in the exponential and propagation regimes using two stabilization parameters. 
Both the steady-state and transient solution are considered. The numerical procedure can be considered as an alternative to the two-parameter stabilized methods proposed in previous works. Some of the advantages of the formulation here proposed are its simplicity and that it can be naturally extended to multidimensional problems.

The stabilized formulation we present is based on the standard Galerkin FEM solution of the modified governing differential equations derived via the FIC method [42, 44, 45, 47, 49, 50].

The steady-state problem is considered first. The Galerkin FIC-FEM formulation introduces a residual-based term into the discretized equations in space which is governed by two stabilization parameters. The optimal value of these parameters ensuring an exact (nodal) FEM solution using uniform meshes of linear 2-noded elements is obtained. In the absence of the absorption term the formulation simplifies to the standard one-parameter Petrov-Galerkin approach for the advectiondiffusion problem. For the diffusion-reaction case one stabilization parameter is just needed and the diffusion-type stabilization term is identical to that obtained by Felippa and Oñate [17] using a variational FIC approach. A procedure for computing the stabilization parameters for the transient problem is proposed. The merit of the new FIC-FEM formulation is that it yields stabilized and accurate numerical solutions for the steady-state and transient 1D advection-diffusion-radiation equation for all the range of physical parameters and boundary conditions.

The lay-out of the paper is the following. In the next section we formulate the FIC form of the equations governing steady-state 1D convection-diffusion-reaction problem. The finite element discretization using the linear 2-noded element is presented. Then the optimal stabilization parameters yielding nodally exact solutions for the sourceless case using a regular mesh of linear elements are obtained. The expression of the optimal stabilization parameters for a constant and a linear source function is presented. The accuracy of the new FIC-FEM formulation for steady-
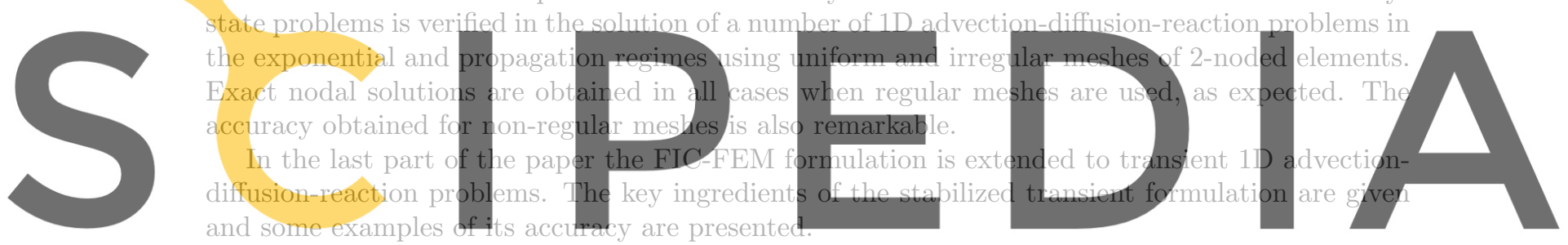

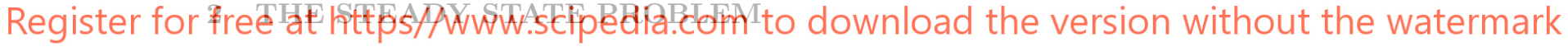

\subsection{Governing equations}

The governing equations for the steady-state convection-diffusion-reaction problem in a 1D domain of length $l$ are

\section{Transport balance}

$$
r_{s}=0 \quad \text { in } \Omega:=[0, l]
$$

with

$$
r_{s}:=\rho c u \frac{d \phi}{d x}-\frac{d}{d x}\left(k \frac{d \phi}{d x}\right)+s \phi-Q
$$

The index $s$ in $r_{s}$ distinguishes the governing differential equation over the domain $\Omega$ for the steady-state case from the transient one studied in Section 8.

\section{Boundary conditions}

$$
\phi-\phi^{p}=0 \quad \text { on } \Gamma_{\phi}
$$




$$
r_{\Gamma}=0 \quad \text { on } \Gamma_{q}
$$

with

$$
r_{\Gamma}=\left(-\rho c u \phi+k \frac{d \phi}{d x}\right) n+q^{p}=0
$$

In Eqs.(1)-(3) $\phi$ is the transported variable (for instance, the temperature in a heat transfer problem), $u$ is the velocity, $\rho, c$ and $k$ are the density, the specific flux parameter and the conductivity of the material, respectively, $s$ is the reaction parameter $(s>0$ is the absorption or dissipation parameter and $s<0$ is the production parameter). In the following, and unless otherwise specified, we will assume that the problem parameters $(u, \rho, c, k, s)$ are constant over the analysis domain.

In Eqs.(2) and (3) $\phi^{p}$ and $q^{p}$ are the prescribed values of the transported variable and the outgoing flux at the Dirichlet and Neumann boundaries $\Gamma_{\phi}$ and $\Gamma_{q}$, respectively, with $\Gamma_{\phi} \cup \Gamma_{q}=\Gamma$, $\Gamma$ being the total boundary of the domain and $n$ defines the sign of the normal direction at the boundary edges. For simplicity, we will assume in this work $\Gamma_{\phi}$ and $\Gamma_{q}$ to be placed at $x=0$ and $x=l$, respectively. Hence, $n=+1$ at $x=l$.

Eqs.(1)-(3) solve the following particular problems:

\section{(i) Advection-diffusion $(s=0)$}

(ii) Helmholtz $(u=0, s<0)$

(iii) Advection-reaction $(k=0)$

(iv) Diffusion-reaction $(u=0)$

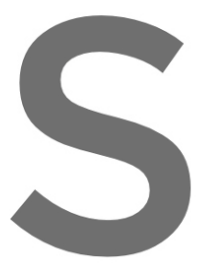

The different equat transport, acoustics, among others.

The exact solution of Eqs.
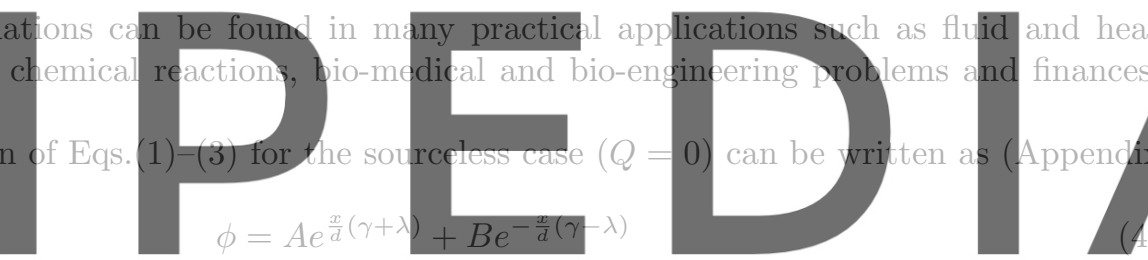

where $A$ and $B$ are coefficients that depend of the boundary conditions, $\lambda$ and $\gamma$ are non dimensional

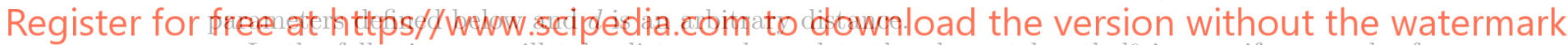

In the following we will take distance $d$ equal to the element length $l^{e}$ in a uniform mesh of linear 2-noded elements. Hence, we can define $\lambda$ as

$$
\lambda=\left(\gamma^{2}+w\right)^{1 / 2}
$$

where

$$
\gamma=\frac{\rho c u l^{e}}{2 k} \quad \text { and } \quad w=\frac{s\left(l^{e}\right)^{2}}{k}
$$

are, respectively, the element Peclet number and a non-dimensional parameter that expresses the relative value of the reaction and diffusion effects.

The exponential and (exponentially modulated) propagating regimes are determined by

$$
\begin{array}{ll}
\gamma^{2}+w \geq 0 & \text { exponential solution } \\
\gamma^{2}+w<0 & \text { propagation solution }
\end{array}
$$

For the advection-reaction case, $k=0$ and then only the upstream boundary condition is necessary. If $u>0$, the exact steady-state solution is

$$
\phi(x)=\phi^{p} e^{-\frac{s}{\rho c u}}
$$




\subsection{Finite Increment Calculus (FIC) expressions}

The governing equations (1)-(3a) are modified following the FIC approach in space [42, 44, 45, $53,54,57]$ as

\section{Transport balance}

$$
r_{s}-\frac{1}{2} h \frac{d r_{s}}{d x}=0 \quad \text { in } \Omega
$$

\section{Boundary conditions}

$$
\begin{gathered}
\phi-\phi^{p}=0 \quad \text { on } \Gamma_{\phi} \\
r_{\Gamma}+\frac{1}{2} h r_{s}=0 \quad \text { on } \Gamma_{q}
\end{gathered}
$$

where $h$ is a characteristic distance that can have a positive or negative value and $r_{S}$ and $r_{\Gamma}$ are defined in Eqs.(1b) and (3b), respectively.

Eqs.(8) and (10) are obtained by expressing the balance of fluxes in an arbitrary segment of finite length $h$ (termed the characteristic length) within the problem domain and at the Neumann boundary, respectively. The variations of the transported variable within the balance segment are approximated by Taylor series expansions retaining one order higher terms than in the infinitesimal theory [42]. The underlined terms in Eqs.(8) and (10) emanate from these series expansions and they are essential to derive stabilized numerical schemes.

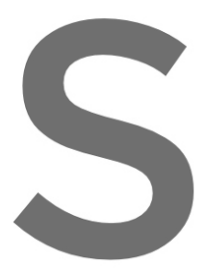

Note that $r_{s} \neq 0$ and $r_{\Gamma} \neq 0$ in the FIC balance equ characteristic length $h$ infinitesimal form,

Similarly as in

depends on the valu the discrete level $h$
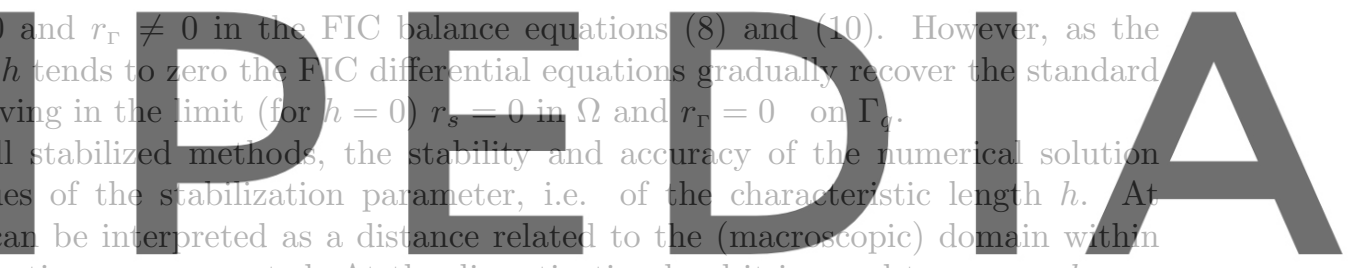

which the space derivatives are computed. At the discretization level it is usual to express $h$ as a

Register for freertion of a typical grid dimengion (i.e. the element length fre 1D FEM problems) [42 the watermark

cal method. It is interesting that many of the stabilized FEM can be recovered using the FIC formulation. The FIC-FEM method has been successfully applied to the finite element solution problems of convection-diffusion [42, 44, 45, 53], diffusion-absorption and Helmholtz [17], advectiondiffusion-absorption [54, 57], incompressible fluid flow [55, 56, 58, 60, 62], fluid-structure-interaction $[47,51,59]$, particulate flows and standard and incompressible solid mechanics [49, 52, 61]. Applications of the FIC stabilization approach to meshless problems solved using the finite point method are reported in $[46,48]$.

Eqs.(8) and (10) hold for any definition of the characteristic distance $h$. Let us now choose two specific forms of $h$, as

$$
\begin{aligned}
h_{u} & =\alpha_{u} l^{e} \\
h_{g} & =2 \alpha_{g} \frac{k}{r_{s}} \phi^{\prime}
\end{aligned}
$$

where the prime superindex denotes the space derivative, and $\alpha_{u}$ and $\alpha_{g}$ are stabilization parameters. In this work we will frequently refer to $\alpha_{u}$ and $\alpha_{g}$ as the streamline (or SUPG) and isotropic diffusion parameters, respectively. Indeed, this distinction is more meaningful in multidimensional problems [55]. 
The choice of $h_{u}$ and $h_{g}$ in Eqs.(11) and (12) leads to a simple form of the stabilization terms, as it will be shown later. The characteristic length vector $h$ is defined as the sum of $h_{u}$ and $h_{g}$, i.e.

$$
h=h_{u}+h_{g}=\alpha_{u} l^{e}+2 \alpha_{g} \frac{k}{r_{s}} \phi^{\prime}
$$

Substituting Eqs.(11) and (12) into (13) and this into (8) gives the FIC governing equations as

$$
r_{s}-\frac{1}{2}\left[\alpha_{u} l^{e}+2 \alpha_{g} \frac{k \phi^{\prime}}{r_{s}}\right] \frac{d r_{s}}{d x}=0
$$

\section{WEIGHTED RESIDUAL FORM}

The weighted residual form is written using the definition of $r_{\Gamma}$ of Eq.(3b) and $n=1$ at $x=l$ as

$$
\int_{0}^{l} W\left(r_{s}-\frac{h}{2} \frac{d r_{s}}{d x}\right) d x+\left[W\left(-\rho c u \phi+k \frac{d \phi}{d x}+q^{P}+\frac{h}{2} r_{s}\right)\right]_{l}=0
$$

where $W$ are appropriate space weighting functions such that $W=0$ on $\Gamma_{\phi}$ (i.e. at $x=0$ ).

Integrating by parts the term involving $h$ in the first integral leads to

$$
\int_{0}^{l} W r_{s} d x+\int_{0}^{l} \frac{h}{2} \frac{d W}{d x} r_{s} d x+\left[W\left(-\rho c u \phi+k \frac{d \phi}{d x}+q^{P}\right)\right]_{l}=0
$$

Note that the boundary term involving $r_{s}$ has vanished in Eq.(16) due to the integration by parts. Also in the derivation of Eq.(16) we have neglected the space derivative of $h$.

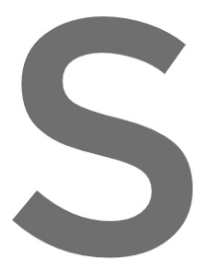

Substituting the and diffusive terms
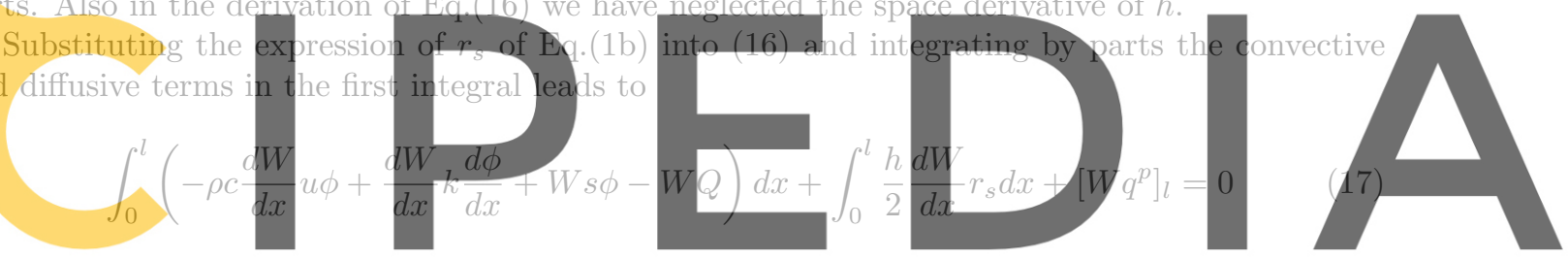

Let us substitute the expression of $h$ of Eq.(13) into the second integral of Eq.(17). Using the

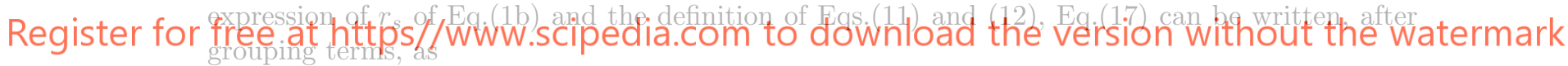

$$
\begin{array}{r}
\int_{0}^{l}\left(-\frac{d W}{d x} \rho c \bar{u} \phi+\frac{d W}{d x} \bar{k} \frac{d \phi}{d x}+W s \phi-W Q\right) d x- \\
-\int_{0}^{l} \frac{d W}{d x} \frac{h_{u}}{2} \frac{d}{d x}\left(k \frac{d \phi}{d x}\right) d x+\left[W q^{p}\right]_{l}=0
\end{array}
$$

where

$$
\begin{aligned}
& \bar{W}=W+\alpha_{u} \frac{l^{e}}{2} \frac{d W}{d x} \\
& \bar{u}=\left(1-\frac{\alpha_{u} s l^{e}}{2 \rho c u}\right) u
\end{aligned}
$$

and

$$
\bar{k}=\left(k+\alpha_{u} \frac{\rho c u l^{e}}{2}+\alpha_{g} k\right)=k+k_{u}+k_{g}
$$

In Eq.(19c) $k_{u}$ and $k_{g}$ are (positive) stabilization diffusion parameters given by

$$
k_{u}=\alpha_{u} \frac{\rho c u l^{e}}{2}=\alpha_{u} \gamma k \quad, \quad k_{g}=\alpha_{g} k
$$


Eq.(19c) can be rewritten as

$$
\bar{k}=k(1+\theta)
$$

with

$$
\theta=\alpha_{u} \gamma+\alpha_{g}
$$

being the generalized stabilization parameter, computed from the contribution of the streamline and isotropic diffusion parameters $\alpha_{u}$ and $\alpha_{g}$.

The term $\theta k$ in Eq.(21) is the so-called artificial (or stabilizing) diffusion that is introduced using different arguments in many stabilized FEM [6, 14, 27, 28, 69].

Remark 1. The velocity $\bar{u}$ in Eq.(19b) can be rewritten as

$$
\bar{u}=\left(1-\alpha_{u} \frac{w}{4 \gamma}\right) u=\left(1-\frac{\alpha_{u}}{2} \sigma\right) u
$$

where $\sigma=\frac{w}{2 \gamma}=\frac{s l^{e}}{\rho c u}$ is the Damköhler number.

The pseudo-Peclet number is defined as

$$
\bar{\gamma}=\frac{\rho c \bar{u} l^{e}}{2 k}=\left(1-\frac{\alpha_{u}}{2} \sigma\right) \gamma
$$

The challenge now is to find the values of the two stabilization parameters $\alpha_{u}$ and $\alpha_{g}$ (or those of the related parameters $\theta$ and $\bar{\gamma}$ ) that lead to a stable and accurate numerical solution. In this work, we will find an optimal expression of these parameters that yields exact nodal solutions

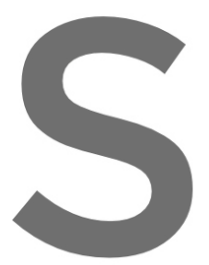
for uniform meshes accurate results for 4 FINITE ELEMF

The transported
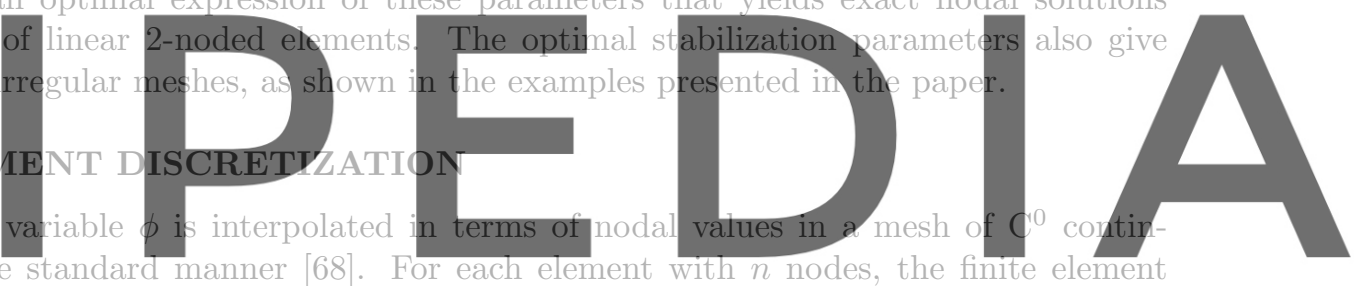

uous elements in the sta

where $N_{i}(\mathbf{x})$ is the space shape function of node $i$ and $\phi_{i}$ is the nodal value of the approximate function $\hat{\phi}$ at the $i$ th node.

Substituting the approximation (25) into the stabilized weak form (17) and choosing a Galerkin weighting $\left(W_{i}=N_{i}\right)[68]$ leads to the following system of discretized equations

$$
[\mathbf{K}-\mathbf{C}+\mathbf{S}] \boldsymbol{\phi}=\mathbf{f}
$$

where vector $\boldsymbol{\phi}=\left[\phi_{1}, \phi_{2}, \cdots, \phi_{N}\right]^{T}$ lists the values of the approximated transported variable at the $N$ nodes in the mesh, $\mathbf{K}$ is the diffusion matrix, $\mathbf{C}$ is the convection matrix, $\mathbf{S}$ is the radiation matrix and $\mathbf{f}$ is the nodal external flux vector. These matrices and vector $\mathbf{f}$ are assembled from the element contributions given by

$$
\begin{aligned}
K_{i j}^{e} & =\int_{l^{e}}\left[\frac{d N_{i}}{d x} \bar{k} \frac{d N_{j}}{d x}-\frac{d N_{i}}{d x} \frac{h_{u}}{2} \frac{d}{d x}\left(k \frac{d N_{j}}{d x}\right)\right] d x \\
C_{i j}^{e} & =\int_{l^{e}} \frac{d N_{i}}{d x} \rho c \bar{u} N_{j} d x \quad, \quad S_{i j}^{e}=\int_{l^{e}} s N_{i} N_{j} d x \\
f_{i}^{e} & =\int_{l^{e}}\left[N_{i}+\alpha_{u} \frac{l^{e}}{2} \frac{d N_{i}}{d x}\right] Q d x
\end{aligned}
$$


Note that the second term in the expression of $K_{i j}^{e}$ in Eq.(27a) vanishes for linear 2-noded elements.

The prescribed outgoing flux $\left(q^{p}\right)$ is directly added (with a negative sign) to the component of vector $\mathbf{f}$ for the node laying on the Neumann boundary, as usual.

\subsection{Element matrices for the linear 2-noded element}

The element matrices in Eq.(26) take the following form for the linear 2-noded element

$$
\mathbf{K}^{e}=\frac{\bar{k}}{l^{e}}\left[\begin{array}{cc}
1 & -1 \\
-1 & 1
\end{array}\right] \quad, \quad \mathbf{C}^{e}=\frac{\rho c \bar{u}}{2}\left[\begin{array}{cc}
-1 & -1 \\
1 & 1
\end{array}\right] \quad, \quad \mathbf{S}^{e}=\frac{s l^{e}}{6}\left[\begin{array}{ll}
2 & 1 \\
1 & 2
\end{array}\right]
$$

The form of vector $\mathbf{f}^{e}$ for 2-noded elements depends on the particular expression of the source term. For a constant source $Q(x)=Q_{0}$,

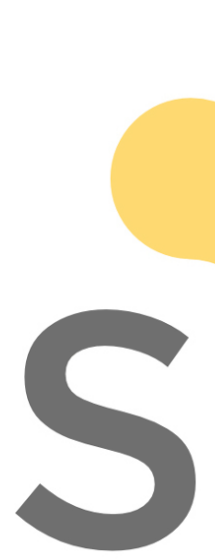

$$
\begin{gathered}
\mathrm{f}^{e}=\frac{Q_{0} l^{e}}{2}\left\{\begin{array}{c}
1-\alpha_{u} \\
1+\alpha_{u}
\end{array}\right\} \\
\alpha_{u}^{e}=\frac{1}{l^{e}} \int_{l^{e}} \alpha_{u} d x
\end{gathered}
$$

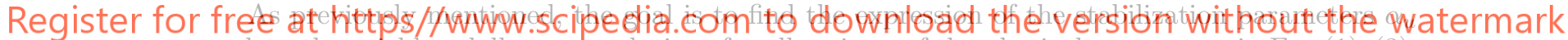 and $\alpha_{g}$ that yield nodally exact solutions for all regimes of the physical parameters in Eqs.(1)-(3). Indeed, this is possible for the linear 2-noded element as it will be shown below.}

Let us consider a steady-state problem with $Q=0$, uniform material properties and Dirichlet boundary conditions with $\phi$ fixed to values 0 and $\phi^{p}$ at $x=0$ and $x=l$, respectively. The problem is solved with a mesh of linear 2-noded elements of equal length $l^{e}$.

The FE equation stencil for three consecutive nodes $i-1, i, i+1$ is

$$
\frac{\rho c \bar{u}}{2}\left(-\phi_{i-1}+\phi_{i+1}\right)+\frac{\bar{k}}{l^{e}}\left(-\phi_{i-1}+2 \phi_{i}-\phi_{i+1}\right)+\frac{s l^{e}}{6}\left(\phi_{i-1}+4 \phi_{i}+\phi_{i+1}\right)=0
$$

Eq.(29) can be rewritten after dividing by $\frac{k}{l^{e}}$ as

$$
\bar{\gamma}\left(-\phi_{i}+\phi_{i+1}\right)+(1+\theta)\left(-\phi_{i-1}+2 \phi_{i}-\phi_{i+1}\right)+\frac{w}{6}\left(\phi_{i-1}+4 \phi_{i}+\phi_{i+1}\right)=0
$$

or

$$
-\left(\bar{\gamma}+1+\theta-\frac{w}{6}\right) \phi_{i-1}+2\left(1+\theta+\frac{w}{3}\right) \phi_{i}-\left(-\bar{\gamma}+1+\theta-\frac{w}{6}\right) \phi_{i+1}=0
$$

where $\bar{\gamma}$ was defined in Eq.(24). 


\subsection{Optimal stabilization parameters}

The exact solution of Eq.(1) for $Q=0$ and uniform properties satisfies the following equation in differences over a grid of equally - spaced segments of length $l^{e}$ (Appendix A)

$$
-e^{\gamma} \hat{\phi}_{i-1}+2 \cosh \lambda \hat{\phi}_{i}-e^{-\gamma} \hat{\phi}_{i+1}=0
$$

with $\lambda$ and $\gamma$ as defined in Eqs.(5) and (6).

In Eq.(32), $(\cdot)$ denotes the exact nodal values of the transported variable.

For convenience, we will obtain first the optimal value of the parameters $\theta$ and $\bar{\gamma}$ that yield exact nodal finite element results, and subsequently that of the related stabilization parameters $\alpha_{u}$ and $\alpha_{g}$.

Assuming nodal exactness of the numerical solution and comparing the coefficients in Eqs.(31) and (32), we deduce the following two equalites

$$
\begin{aligned}
& \frac{\bar{\gamma}+1+\theta-\frac{w}{6}}{2\left(1+\theta+\frac{w}{3}\right)}=\frac{e^{\gamma}}{2 \cosh \lambda} \\
& \frac{-\bar{\gamma}+1+\theta-\frac{w}{6}}{2\left(1+\theta+\frac{w}{3}\right)}=\frac{e^{-\gamma}}{2 \cosh \lambda}
\end{aligned}
$$

Solution of Eqs.(33) and (34) for $\theta$ and $\bar{\gamma}$ gives
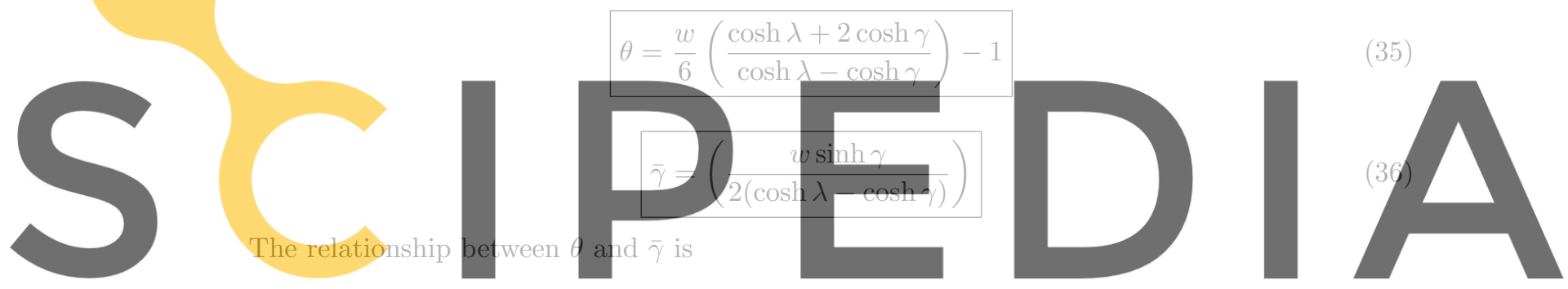

$$
\theta=\bar{\gamma} \operatorname{coth} \gamma+\frac{w}{6}-1
$$

(37)

Register for free at https//www.scipedia.com to download the version without the watermark

Let us obtain now the expression of the two stabilization parameters $\alpha_{u}$ and $\alpha_{g}$.

From Eq.(36) and the definition of $\bar{\gamma}$ (Eq.(24)) and the expression of $\bar{u}$ of Eq.(23) we deduce

$$
\alpha_{u}(\gamma, w)=\frac{4 \gamma}{w}-\frac{2 \sinh \gamma}{\cosh \lambda-\cosh \gamma}
$$

It can be readily found

$$
\begin{aligned}
& \lim _{\gamma \rightarrow 0} \alpha_{u}(\gamma, w)=\alpha_{u}(w)=0 \\
& \lim _{w \rightarrow 0} \alpha_{u}(\gamma, w)=\alpha_{u}(\gamma)=\operatorname{coth} \gamma-\frac{1}{\gamma}
\end{aligned}
$$

Expression (39b) is the optimal stabilization parameter for the $1 \mathrm{D}$ convection-diffusion case problem solved with the SUPG method and 2-noded elements [14, 69]. Note that $\alpha_{u}(\gamma) \rightarrow 0$ for $\gamma \rightarrow 0$ and $\alpha_{u}(\gamma) \rightarrow 1$ for $\gamma \rightarrow \infty$.

From the definition of $\theta$ (Eq.(22)) and using Eqs.(35) and (38) we obtain

$$
\alpha_{g}(\gamma, w)=\frac{\frac{w}{6}(\cosh \lambda+2 \cosh \gamma)+2 \gamma \sinh \gamma}{\cosh \lambda-\cosh \gamma}-\frac{4 \gamma^{2}}{w}-1
$$


From Eq.(40) we can find

$$
\begin{gathered}
\lim _{w \rightarrow 0} \alpha_{g}(\gamma, w)=\alpha_{g}(\gamma)=0 \\
\lim _{\gamma \rightarrow 0} \alpha_{g}(\gamma, w)=\alpha_{g}(w)=\frac{w}{4 \sinh ^{2}\left(\frac{\sqrt{w}}{2}\right)}+\frac{w}{6}-1
\end{gathered}
$$

Expression (42) is the optimal stabilization parameter for the 1D diffusion-reaction problem solved with the FIC-FEM approach using 2-noded elements. This expression was obtained by Oñate et al. [54].

The reaction stabilization parameter $\alpha_{g}$ of Eq.(42) can be expressed as

$$
\alpha_{g}(w)=\left[\frac{1}{4 \sinh ^{2}\left(\frac{\sqrt{w}}{2}\right)}+\frac{1}{6}-\frac{1}{w}\right] w=\bar{\alpha}_{g}(w) w
$$

with

$$
\bar{\alpha}_{g}(w)=\frac{1}{4 \sinh ^{2}\left(\frac{\sqrt{w}}{2}\right)}+\frac{1}{6}-\frac{1}{w}
$$

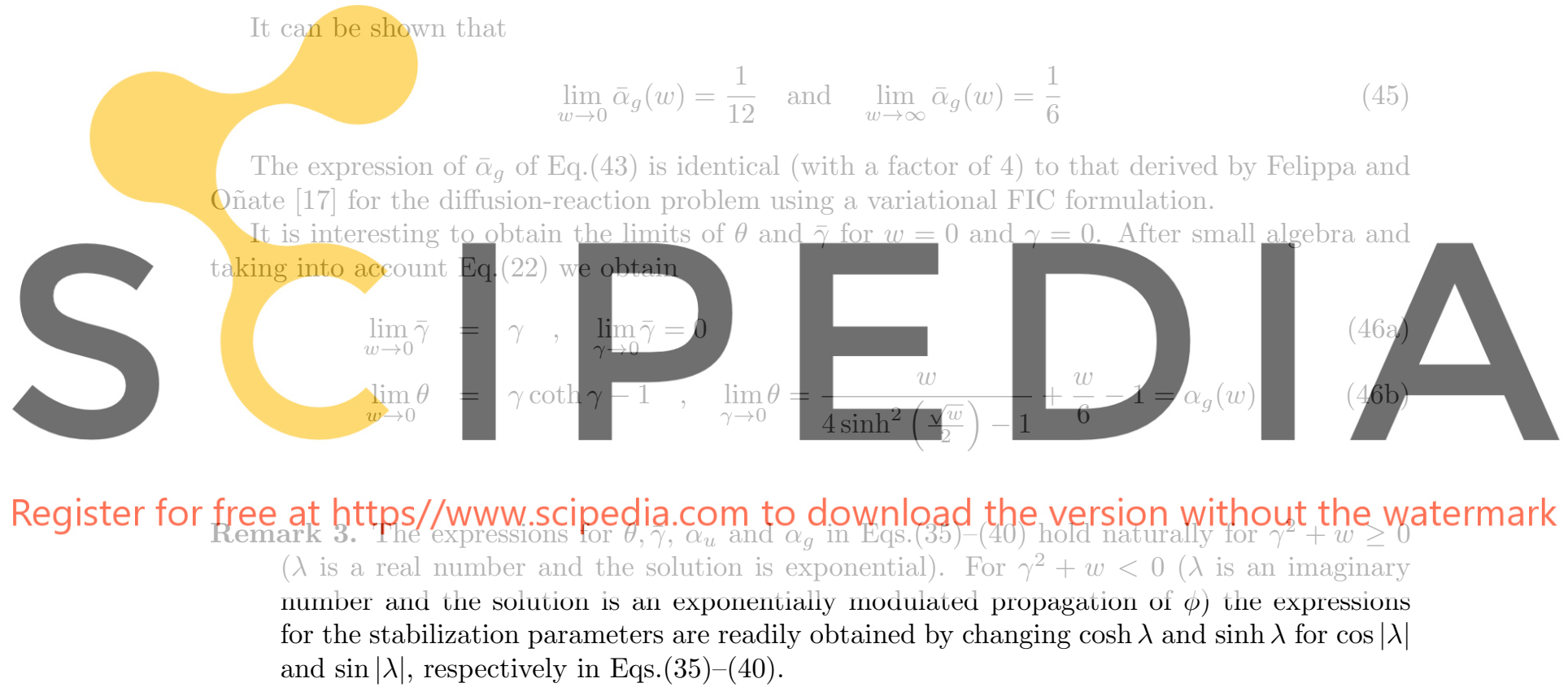

Remark 4. It can be verified that $\alpha_{g}$ is greater or equal to zero for all values of $\gamma$ and $w$. This guarantees that the stabilization diffusion $k_{g}$ is positive (see Eq.(20)).

Remark 5. The expression of $\alpha_{u}$ satisfies the condition $\operatorname{sgn}\left(\alpha_{u}\right)=\operatorname{sgn}(\gamma)$, where $\operatorname{sgn}(\cdot)$ is the sign function. This ensures that $\alpha_{u} \gamma \geq 0$ and guarantees that the stabilization diffusion $k_{u}$ is positive (Eq.(20)). As $k_{g}$ is also positive (Remark 4) the stabilization parameter $\theta$ is always positive (Eq.(22)).

Remark 6. Substituting Eq.(38) into (24) it is deduced that $\operatorname{sgn}(\bar{\gamma})=\operatorname{sgn}(\gamma)$. Consequently, $\bar{\gamma} \operatorname{coth} \gamma \geq|\bar{\gamma}|$ and, therefore, the generalized stabilized parameter $\theta$ satisfies the general conditions for stability of the convection-diffusion-radiation problem (Appendix C), i.e.

$$
\theta>\frac{w}{6}+|\bar{\gamma}|-1
$$

The proof of Eq.(47) is straightforward using the expression of $\theta$ of Eq.(37). 
Remark 7. The expressions of $\alpha_{u}$ and $\alpha_{g}$ can be written in more compact form in terms of the Damköhler number $\sigma$ as

$$
\begin{gathered}
\alpha_{u}=\frac{2}{\sigma}\left(1-\frac{\sigma \tanh \gamma}{\xi-1}\right) \\
\alpha_{g}=\gamma\left[\frac{\sigma}{3}\left(\frac{\xi+2}{\xi-1}\right)-\alpha_{u}\right]
\end{gathered}
$$

with $\xi=\frac{\cosh \lambda}{\cosh \gamma}$.

\section{EXTENSION TO THE SOURCE CASE $(Q \neq 0)$}

\subsection{General expressions}

The exact solution of Eq.(1) for $Q \neq 0$ satisfies the following equation in differences in a grid of equally-spaced segments of length $l^{e}$ (Appendix B)

$$
-e^{\gamma} \hat{\phi}_{i-1}+2 \cosh \lambda \hat{\phi}_{i}-e^{-\gamma} \hat{\phi}_{i+1}=-e^{\gamma} \phi_{i-1}^{p}+2 \cosh \lambda \phi_{i}^{p}-e^{-\gamma} \phi_{i+1}^{p}
$$

where $\phi_{i-1}^{p}, \phi_{i}^{p}$ and $\phi_{i+1}^{p}$ are the values of the particular solution $\phi^{p}(x)$ of the differential equations (1) computed at three consecutive nodes $i-1, i$ and $i+1$, respectively.

The FE stencil in a mesh of equal length 2-noded elements accounting for the external flux term can be written as

$$
-\left(\bar{\gamma}+1+\theta-\frac{w}{6}\right) \phi_{i-1}+2\left(1+\theta+\frac{w}{3}\right) \phi_{i}-\left(-\bar{\gamma}+1+\theta-\frac{w}{6}\right) \phi_{i+1}=\frac{\left(l^{e}\right)^{2}}{k}\left(1+\alpha_{i}\right) f_{i}
$$

where $f_{i}$ is the external flux at node $i$, neglecting the stabilization terms (i.e. making $\alpha_{u}=0$ in the expression of $\mathbf{f}^{e}$ of Eq. $\left.(27 \mathrm{c})\right)$ and $\alpha_{i}$ is a nodal stabilization parameter that results from the assembly of the stabilization terms in vector $\mathbf{f}^{e}$.

The value of $\alpha_{i}$ can be obtained by equalling the r.h.s. of Eqs.(49) and (50) after dividing by the coefficient multiplying $\phi_{i}$ and $\hat{\phi}_{i}$ in each of the two equations. This gives

$$
\frac{\left(l^{e}\right)^{2}\left(1+\alpha_{i}\right) f_{i}}{2 k\left(1+\theta+\frac{w}{3}\right)}=\frac{-e^{\gamma} \phi_{i-1}^{p}+2 \cosh \lambda \phi_{i}^{p}-e^{-\gamma} \phi_{i+1}^{p}}{2 \cosh \lambda}
$$

From Eq.(51) we find

$$
\alpha_{i}=-1+\frac{k\left(1+\theta+\frac{w}{3}\right)\left(-e^{\gamma} \phi_{i-1}^{p}+2 \cosh \lambda \phi_{i}^{p}-e^{-\gamma} \phi_{i+1}^{p}\right)}{\left(l^{e}\right)^{2} f_{i} \cosh \lambda}
$$

In the following lines we compute the optimal value of $\alpha_{i}$ for different external source terms $Q(x)$ in a uniform mesh of 2-noded elements.

\subsection{Constant external flux: $Q(x)=Q_{0}$}

The particular solution of Eq.(1a) is

$$
\phi^{p}(x)=\frac{Q_{0}}{s}
$$

On the other hand, the value of the nodal flux is deduced from Eq.(27b) as

$$
f_{i}=Q_{0} l^{e}
$$

Substituting Eqs.(53) and (54) into (52) gives after small algebra

$$
\alpha_{i}=-1+\frac{2(\cosh \lambda-\cosh \gamma)}{w \cosh \lambda}\left(1+\theta+\frac{w}{3}\right)
$$


Substituting the expression of $\theta$ of Eq.(35) into (55) yields

$$
\alpha_{i}=0
$$

Hence, nodally exact solutions will be obtained for a constant source and a mesh of equal length 2-noded elements neglecting the effect of the stabilization term (i.e. $\alpha_{u}=0$ ) in the expression of $\mathbf{f}^{e}$ of Eq.(28b).

This result is consistent with the fact that the contribution of the stabilization term in the nodal flux vector vanishes for the interior nodes in the assembled expression of $\mathbf{f}$ for this case, as explained in Remark 2.

\subsection{Linear source term: $Q(x)=a x+b$}

The particular solution of Eq.(4) is

$$
\phi^{p}(x)=\frac{a}{s} x-\left(\frac{u}{s^{2}} a-\frac{b}{s}\right)
$$

The nodal flux term is deduced from Eq.(27b) as

$$
f_{i}=b+\frac{a}{2}\left(1+2 x_{i}-l^{e}\right)
$$

Substituting Eqs.(57) and (58) into (52) gives the optimal value of $\alpha_{i}$ as

$$
\begin{array}{r}
\alpha_{i}=-1+\frac{k\left(1+\theta+\frac{w}{3}\right)}{\left(l^{e}\right)^{2}\left(b+\frac{a}{2}\left(1+2 x_{i}-l^{e}\right)\right) l^{e} \cosh \lambda} \times \\
\times\left[2(\cosh \lambda-\cosh \gamma)\left(\frac{b}{s}-\frac{u}{s^{2}} a\right)+\left(-e^{\gamma} x_{i-1}+2 \cosh \lambda x_{i}-e^{-\gamma} x_{i+1}\right) \frac{a}{s}\right]
\end{array}
$$

Noting that $x_{i-1}=x_{i}-l^{e}$ and $x_{i+1}=x_{i}+l^{e}$ yields

$$
\alpha_{i}=-1+\frac{1+\theta+\frac{w}{3}}{\left(b+\frac{a}{2}\left(1+2 x_{i}-l^{e}\right)\right) \cosh \lambda}\left[\left(\frac{2\left(b+a x_{i}\right)}{w}-\frac{4 \gamma a l^{e}}{w^{2}}\right)(\cosh \lambda-\cosh \gamma)+\frac{2 a l^{e}}{w} \sinh \gamma\right]
$$

Hence, nodally exact solutions will be obtained for a linear source term and a regular mesh of linear 2-noded elements using the optimal stabilization parameters obtained in Section 5 and inserting the value of $\alpha_{i}$ of Eq.(60) into Eq.(50) with $\theta$ given by Eq.(35).

Similar expressions for the optimal value of $\alpha_{i}$ can be found for other external source terms $Q(x)$ following the same procedure.

\section{CONVECTION-RADIATION PROBLEM}

The governing equations for the convection-radiation problem are

$$
r:=\rho c u \frac{d \phi}{d x}+s \phi-Q=0
$$

with

$$
\begin{gathered}
\phi-\phi^{p}=0 \quad \text { on } \quad \Gamma_{\phi} \\
-\rho c u \phi n+q^{p}=0 \quad \text { on } \quad \Gamma_{q}
\end{gathered}
$$

The FE stencil for the sourceless case is deduced from Eq.(31) substituting the expression of $\bar{\gamma}$ of Eq.(24) and then after multiplying by $k$ and subsequently making $k=0$. The resulting expression is

$$
\begin{aligned}
-\left[\left(1-\frac{\alpha_{u}}{2} \sigma+\alpha_{u}\right) \frac{\rho c u l}{2}+\left\langle\alpha_{g} k\right\rangle-\frac{s l^{2}}{6}\right] \phi_{i-1}+2\left[\alpha_{u} \frac{\rho c u l}{2}+\left\langle\alpha_{g} k\right\rangle-\frac{s l^{2}}{3}\right] \phi_{i}- \\
{\left[-\left(1-\frac{\alpha_{u}}{2} \sigma-\alpha_{u}\right) \frac{\rho c u l}{2}+\left\langle\alpha_{g} k\right\rangle-\frac{s l^{2}}{6}\right] \phi_{i+1}=0 }
\end{aligned}
$$


where $\left\langle\alpha_{g} k\right\rangle$ denotes the new stabilization parameter for the case of zero diffusion.

The analytical solution of Eq.(61) is

$$
\phi=A e^{-\frac{s}{\rho c u} x}=A e^{-\frac{\sigma}{l^{e}} x}
$$

From Eq.(64) we deduce for a uniform mesh of 2-noded element with $x_{i-1}=x_{i}-l^{e}$ and $x_{i+1}=x_{i}+l^{e}$

$$
\phi_{i}=A e^{-\frac{\sigma}{l e} x_{i}} \quad, \quad \phi_{i+1}=e^{-\sigma} \phi_{i} \quad, \quad \phi_{i-1}=e^{\sigma} \phi_{i}
$$

From the relationships in Eq.(65) we can infer that the exact solution of the convection-reaction problem satisfies the following generic stencil

$$
-c_{1} e^{-\sigma} \hat{\phi}_{i-1}+\left(c_{1}+c_{2}\right) \hat{\phi}_{i}-c_{2} e^{\sigma} \hat{\phi}_{i+1}=0
$$

Comparing the stencils in Eq.(63) and (66) we get

$$
\frac{B_{1}}{c_{1} e^{-\sigma}}=\frac{B_{2}}{c_{1}+c_{2}}=\frac{B_{3}}{c_{2} e^{\sigma}}
$$

where

$$
\begin{aligned}
& B_{1}=\left[\left(1-\frac{\alpha_{u}}{2} \sigma+\alpha_{u}\right) \frac{\rho c u l}{2}+\left\langle\alpha_{g} k\right\rangle-\frac{s l^{2}}{6}\right] \phi_{i-1} \\
& B_{2}=2\left[\alpha_{u} \frac{\rho c u l}{2}+\left\langle\alpha_{g} k\right\rangle-\frac{s l^{2}}{3}\right] \phi_{i} \\
& B_{3}=\left[-\left(1-\frac{\alpha_{u}}{2} \sigma-\alpha_{u}\right) \frac{\rho c u l}{2}+\left\langle\alpha_{g} k\right\rangle-\frac{s l^{2}}{6}\right] \phi_{i+1}
\end{aligned}
$$

From Eq.(67) we can obtain a system of two equations to solve for $\alpha_{u}$ and $\left\langle\alpha_{g} k\right\rangle$. After some algebra we get

$$
\begin{aligned}
& \alpha_{u}=\frac{2}{\sigma}\left[1-\frac{\left(c_{1}-c_{2} e^{2 \sigma} \sigma\right.}{\left(c_{1}+c_{2}\right) e^{\sigma}-\left(c_{1}+c_{2} e^{2 \sigma}\right)}\right] \\
& \frac{2\left\langle\alpha_{g} k\right\rangle}{\rho c u l}=\frac{\sigma}{3}\left[\frac{2\left(c_{1}+c_{2} e^{2 \sigma}\right)+\left(c_{1}+c_{2}\right) e^{\sigma}}{\left(c_{1}+c_{2}\right) e^{\sigma}-\left(c_{1}+c_{2} e^{2 \sigma}\right.}\right]-\alpha_{u}
\end{aligned}
$$

As the choice of $c_{1}$ and $c_{2}$ is arbitrary we can get infinite solutions for the stabilization parameters given the characteristic solution of the convection-reaction problem. This is no surprise as it is an under-determined problem. Consider the choice $c_{1} \neq 0$ and $c_{2}=0$. We get

$$
\alpha_{u}=\frac{2}{\sigma}\left[1-\frac{\sigma}{\operatorname{sgn}(u) \sigma-1}\right] \quad, \quad\left\langle\alpha_{g} k\right\rangle=\frac{s\left(l^{e}\right)^{2}}{6}+\frac{\rho c u l^{e}}{2}\left[\frac{\sigma}{\operatorname{sgn}(u) \sigma-1}-\alpha_{u}\right]
$$

Expressions (70) yield the exact nodal solution for the sourceless convection-radiation problem using a mesh of equal length linear 2-noded elements. These expressions also lead to accurate nodal results for a constant source.

For $k=0$ the following expression is obtained from of Eqs.(22) and (70)

$$
\theta k=\alpha_{u} \gamma k+\left\langle\alpha_{g} k\right\rangle=\frac{\rho c u l}{2}
$$

which is the standard result of the SUPG scheme $[6,13,69]$. 


\section{STABILIZED 1D TRANSIENT SOLUTION}

\subsection{Discretized form of the stabilized transient problem}

The governing equation for the transient convection-diffusion-reaction problem is

$$
r_{t}=0 \quad \text { in } \Omega:=[0, l]
$$

with

$$
r_{t}:=\rho c\left(\frac{\partial \phi}{\partial t}+u \frac{\partial \phi}{\partial x}\right)-\frac{\partial}{\partial x}\left(k \frac{\partial \phi}{\partial x}\right)+s \phi-Q
$$

where $\phi(x, t)$ is a function of the space coordinate $x$ and the time $t$.

The boundary conditions are identical to Eqs.(2)-(3) simply changing $k \frac{d}{d x}$ by $k \frac{\partial}{\partial x}$ in Eq.(3b).

The definition of the problem is completed with the initial condition

$$
\phi\left(x, t_{0}\right)=\phi_{0}(x)
$$

where $\phi_{0}(x)$ is the value of the transported variable at time $t=t_{0}$.

The FIC form of the governing equations in the domain $\Omega$ and at the boundary $\Gamma_{q}$ is written as

$$
\begin{gathered}
r_{t}-\frac{1}{2} h \frac{\partial r_{t}}{\partial x}=0 \quad \text { in } \Omega:=[0, l] \\
r_{\Gamma}+\frac{1}{2} h r_{t}=0 \quad \text { on } \Gamma_{q}
\end{gathered}
$$

where $r_{\Gamma}$ is given in Eq.(3b).

The characteristic distance $h$ is now defined as

$$
h=\underbrace{\alpha_{u} l^{e}}_{h_{u}}+\underbrace{2 \alpha_{g} k \frac{\operatorname{sgn}\left(r_{t}\right)}{\left|r_{s}\right|} \phi^{\prime}}_{h_{g}}
$$

Note that Eq.(76) is an extension of Eq.(14).

The weighted residual form in space is derived following the procedure described in Section 3 , as

$$
\int_{0}^{l} W\left(r_{t}-\frac{h}{2} \frac{\partial r_{t}}{\partial x}\right) d x+\left[W\left(r_{\Gamma}+\frac{1}{2} h r_{t}\right)\right]_{l}=0
$$

where $W$ are space weighting functions that vanish on $\Gamma_{\phi}$.

Integrating by parts the integral in Eq.(77) gives

$$
\int_{0}^{l} W r_{t} d x+\int_{0}^{l} \frac{h}{2} \frac{\partial W}{\partial x} r_{t} d x+\left[W r_{\Gamma}\right]_{l}=0
$$

Substituting the expression of $r_{t}$ of Eq.(72b) and integrating by parts the convective and diffusive terms gives, after substituting the expression of $h$ of Eq.(76),

$$
\begin{array}{r}
\int_{0}^{l}\left[\rho c \bar{W} \frac{\partial \phi}{\partial t}-\frac{\partial W}{\partial x} \rho c \bar{u} \phi+\left(k+k_{u}+k_{g} \frac{\left|r_{t}\right|}{\left|r_{s}\right|}\right) \frac{\partial W}{\partial x} \frac{\partial \phi}{\partial x}+W s \phi-\bar{W} Q\right] d x- \\
-\int_{0}^{l} \frac{d W}{d x} \frac{h_{u}}{2} \frac{\partial}{\partial x}\left(k \frac{\partial \phi}{\partial x}\right) d x+\left[W q^{p}\right]_{l}=0
\end{array}
$$

where $\bar{W}, \bar{u}, k_{u}$ and $k_{g}$ are defined in Eqs.(19a), (19b) and (20), respectively.

Note that Eq.(79) coincides with (18) for the steady-state (i.e. when $r_{t} \equiv r_{s}$ ) 
Let us choose a linear finite element interpolation over 2-noded elements as

$$
\phi(x, t) \simeq \hat{\phi}(x, t)=\sum_{i=1}^{2} N_{i}(x) \phi_{i}(t)
$$

where $N_{i}(x)$ are the standard linear functions defined in the space domain and $\phi_{i}(t)$ is the nodal value of the approximate function $\hat{\phi}(x, t)$ at time $t$.

Substituting the approximation (80) into Eq.(79) and choosing a Galerkin weighting, gives the following system of discretized equations in space

$$
\mathbf{M} \dot{\phi}+(\mathbf{K}-\mathbf{C}+\mathbf{S}) \boldsymbol{\phi}=\mathbf{f}
$$

where $\dot{\boldsymbol{\phi}}=\frac{\partial}{\partial t} \boldsymbol{\phi}$ and $\boldsymbol{\phi}$ is the vector of nodal variables (Eq.(26)). The element expression of the matrices and vectors in Eq.(81) is

$$
\begin{aligned}
\mathbf{M}^{e} & =\rho c\left(\frac{l^{e}}{6}\left[\begin{array}{ll}
2 & 1 \\
1 & 2
\end{array}\right]+\frac{\alpha_{u} l^{e}}{2}\left[\begin{array}{cc}
-1 & -1 \\
1 & 1
\end{array}\right]\right) \\
\mathbf{K}^{e} & =\frac{1}{l^{e}}\left(k+k_{u}+k_{g} \int_{l^{e}} \frac{\left|\hat{r}_{t}\right|}{\left|\hat{r}_{s}\right|} d x\right)\left[\begin{array}{cc}
1 & -1 \\
-1 & 1
\end{array}\right]
\end{aligned}
$$

and $\mathbf{C}^{e}, \mathbf{S}^{e}$ and $\mathbf{f}^{e}$ are given by Eqs.(27a), (27b) and (27c), respectively. Also we have assumed that $k, k_{u}$ and $k_{g}$ are constant within an element and the last two are given by Eq.(20).

In Eq. (83), $\hat{r}_{t}=r_{t}(\hat{\phi})$ and $\hat{r}_{s}=r_{s}(\hat{\phi})$ are the transient and steady-state discrete residuals, respectively.

We note that the term involving $\alpha_{u}$ in Eq.(81) vanishes after assembly for regular meshes of equal length elements (except at the boundaries).

Matrix $\mathbf{K}^{e}$ is non linear as it involves the integral of the ratio of the modulus of the transient and steady-state discrete residuals. This term can be computed explicitly as shown in Appendix D. As a consequence of this non-linearity, the system of assembled equations (81) has to be solved iteratively within each time step.

\subsection{Transient solution scheme}

We discretize in time the system of equations (81) using a Generalized Trapezoidal rule [13, 68] and a simple Picard iteration scheme as

$$
\left(\frac{1}{\delta \Delta t} \mathbf{M}+{ }^{i} \mathbf{K}-\mathbf{C}+\mathbf{S}\right)^{i+1} \boldsymbol{\phi}^{n+\delta}={ }^{i} \mathbf{f}^{n+\delta}+\frac{1}{\delta \Delta t} \mathbf{M} \boldsymbol{\phi}^{n}
$$

where $\delta$ is a non dimensional time parameter such that $0.5<\delta \leq 1$ for the integration scheme to be stable $[13,68,69]$ and ${ }^{i}(\cdot)$ denotes values for the $i$ th iteration.

The solution ${ }^{i+1} \boldsymbol{\phi}^{n+1}$ for the $i$ th iteration can be found in terms of ${ }^{i+1} \boldsymbol{\phi}^{n+\delta}$ and $\boldsymbol{\phi}^{n}$ as

$$
{ }^{i+1} \boldsymbol{\phi}^{n+1}=\frac{1}{\delta}{ }^{i+1} \boldsymbol{\phi}^{n+\delta}+\left(1-\frac{1}{\delta}\right) \boldsymbol{\phi}^{n}
$$

The iterations proceed until convergence of the solution for $\boldsymbol{\phi}^{n+1}$ measured in the $L_{2}$ norm is achieved. In the transient problems solved in this work convergence within each time step was typically achieved in 2-3 iterations pure advection cases and 6-8 iterations for convection-diffusionreaction cases.

\subsection{Computation of the stabilization parameters}

The optimal value of the stabilization parameters $\alpha_{u}$ and $\alpha_{g}$ giving nodally exact solutions is quite difficult in the transient case due to the multiple forms that the solution can take as it 
evolves in time. We present a procedure for computing a quasi-optimal value of $\alpha_{u}$ and $\alpha_{g}$ that has proved to yield accurate results for solutions involving the pure advection of a discontinuous function, as well as for transient convection-diffusion-reaction problems evolving towards a steady state solution.

The transient equation $(72 \mathrm{~b})$ can be written in the following form

$$
\rho c u \frac{\partial \phi}{\partial x}-\frac{\partial}{\partial x}\left(k \frac{\partial \phi}{\partial x}\right)+\bar{s} \phi-Q=0
$$

where

$$
\bar{s}=s+s_{t} \quad \text { with } s_{t}=\rho c \frac{\dot{\phi}}{\phi}
$$

Eq.(86) defines a pseudo-stationary problem where a non linear reaction term $s_{t}$ has been introduced.

The non linear reaction term $s_{t}$ can be approximated as

$$
s_{t}=\frac{\rho c}{\delta \Delta t} f(\kappa)
$$

with

$$
f(\kappa) \geq 0 \quad \text { and } \quad \kappa=\frac{\phi^{n+\delta}-\phi^{n}}{\phi^{n+\delta}}
$$

From Eq.(87) we can define an equivalent Damköhler number $\bar{\sigma}$ as

$$
\bar{\sigma}=\frac{\bar{s} l^{e}}{\rho c u}=\sigma+\sigma_{t} \quad \text { with } \quad \sigma_{t}=\frac{1}{\delta C} f(\kappa)
$$

where $\sigma$ is the standard Damköhler number and $C=\frac{u \Delta t}{l^{e}}$ is the element Courant number.

Function $f(\kappa)$ should be designed so that $f(\kappa)=0$ (and $\sigma_{t}=0$ ) for a steady-state problem (or in zones where $\dot{\phi}=0$ ), and $f(\kappa)=2$ (and $\sigma_{t}=\frac{2}{\delta C}$ ) for cases when $\phi(x, t)$ suddenly changes from $\phi^{n}=0$ to a finite value $\phi^{n+\delta}=\phi_{c}$ at a node. The later situation happens, for instance, in the propagation of a step function defined by $\phi_{0}(x)=\phi_{c}$ in a subdomain of $\Omega$ and $\phi_{0}(x)=0$ elsewhere.

Taking these considerations into account, the following definition for $f(\kappa)$ has been chosen

$$
f(\kappa)=2 \tanh \left(\beta \frac{\left|\phi^{n+\delta}-\phi^{n}\right|_{\infty}^{e}}{\left|\phi^{n+\delta}+\phi^{n}\right|_{\infty}^{e}}\right)
$$

where $|a|_{\infty}^{e}$ denotes the maximum value of $a$ within an element and $\beta$ is a (relatively large) positive number that controls the slope of the function $\tanh (\cdot)$ that ranges from zero to one. Very good results were obtained in the transient problems solved in this work using Eq.(91) with $\beta=300$. In this case, the optimal choice of $\beta$ in terms of the nature of the transient solution is a matter that deserves further research.

The stabilization parameters $\alpha_{u}$ and $\alpha_{g}$ for the transient convection-diffusion-radiation problem are then estimated as follows

1. The parameter $\alpha_{u}$ is computed using Eq.(48a) using the value of $\bar{\sigma}$ of Eq.(90) as

$$
\alpha_{u}=\frac{2}{\bar{\sigma}}\left(1-\frac{\bar{\sigma} \tanh \gamma}{\xi-1}\right)
$$

2. The parameter $\alpha_{g}$ is computed in terms of $\gamma$ and $\sigma$ using Eq.(48b) with the value of $\alpha_{u}$ given by Eq.(92),i.e.

$$
\alpha_{g}=\gamma\left[\frac{\sigma}{3}\left(\frac{\xi+2}{\xi-1}\right)-\alpha_{u}\right]
$$



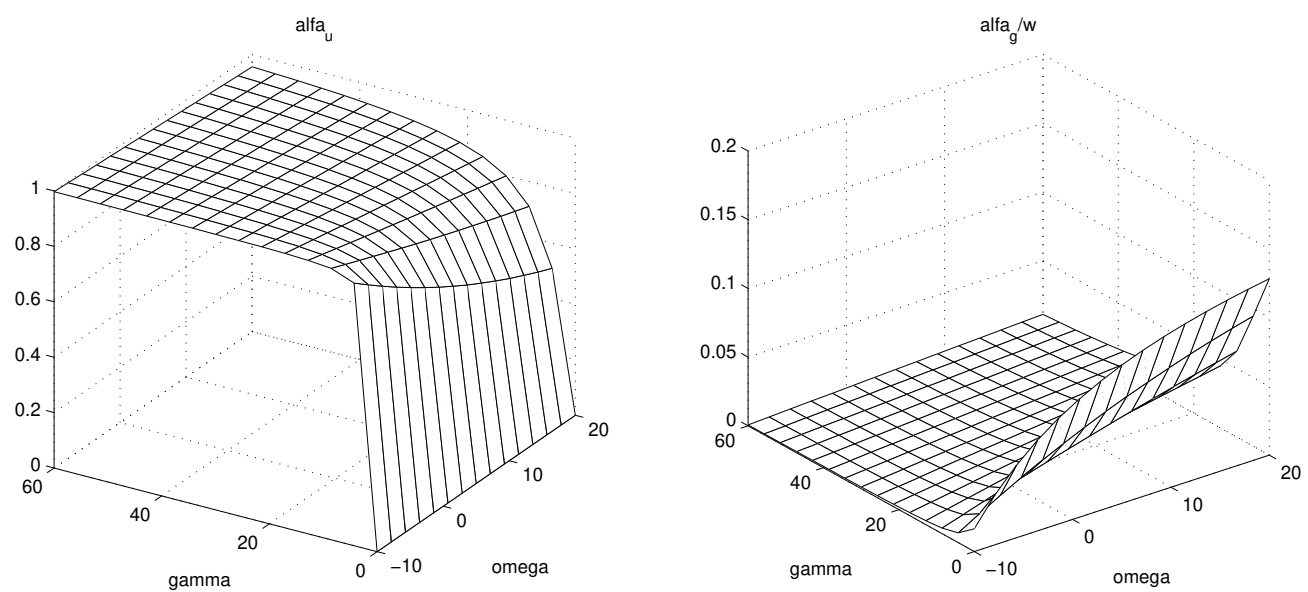

Figure 1: Distribution of $\alpha_{u}$ and $\frac{\alpha_{g}}{w}$ with $\gamma$ and $\omega$

In this manner the effect of the transient term is directly taken into account in the expression of $\alpha_{u}$ via $\sigma_{t}$ and, indirectly, in the expression of $\alpha_{g}$ via $\alpha_{u}$.

Remark 8. A cut-off value is set up for the denominator in Eq.(91) so that $\left|\phi^{n+\delta}+\phi^{n}\right|_{\infty}^{e}=\epsilon$ when $\left|\phi^{n+\delta}+\phi^{n}\right|_{\infty}^{e}<\epsilon$ where $\epsilon$ is a small number. In our computations we have taken $\epsilon=10^{-5}$.

\subsection{Particularization for the transient convection-radiation case}

Following the procedure explained in the previous section and Eqs.(70), we propose the following expressions for computing the stabilization parameters $\alpha_{u}$ and $\left\langle\alpha_{g} k\right\rangle$ for the convection-radiation problem $(k=0)$

$$
\begin{gathered}
\alpha_{u}=\frac{2}{\bar{\sigma}}\left(1-\frac{\bar{\sigma}}{e^{\operatorname{sgn}(u) \bar{\sigma}}-1}\right) \\
\left\langle\alpha_{g} k\right\rangle=\frac{s\left(l^{e}\right)^{2}}{6}+\frac{\rho c u l^{e}}{2}\left[\frac{\sigma}{e^{\operatorname{sgn}(u) \sigma}-1}-\alpha_{u}\right]
\end{gathered}
$$

with $\bar{\sigma}$ given by Eq.(90).

\subsection{Particularization for the pure convection problem}

Numerical algorithms for the time integration of the pure convective transport of a function typically suffer from phase and amplitude errors. For discontinuous functions additional instabilities in the form of numerical wiggles are prone to occur at the vicinity of the interface. The introduction of a stabilization diffusion eliminates the oscillation but can smear the solution near the interface. The derivation of numerical methods that provide stable and accurate solutions is a challenge and several efforts have been reported in the past years [39, 40].

The stabilization parameters $\alpha_{u}$ and $\left\langle\alpha_{g} k\right\rangle$ for this problem are obtained by making $s=0$ in Eqs.(94) and (95). This gives

$$
\alpha_{u}=\frac{2}{\sigma_{t}}\left(1-\frac{\sigma_{t}}{e^{s g n(u) \sigma_{t}}-1}\right) \quad \text { and } \quad\left\langle\alpha_{g} k\right\rangle=\frac{\rho c u l^{e}}{2}\left(1-\alpha_{u}\right)
$$

Let us compute the expression of $\alpha_{u}$ and $\left\langle\alpha_{g} k\right\rangle$ for some representative cases. 
a) Steady-state problem, or $\dot{\phi}=0$ locally, $\left(\sigma_{t}=0\right)$

$$
\lim _{\sigma_{t} \rightarrow 0} \alpha_{u}=1 \quad \text { and } \quad\left\langle\alpha_{g} k\right\rangle=0
$$

b) Sudden jump of $\phi(x, t)$ at a node $\left(\sigma_{t}=\frac{2}{\delta C}\right)$

b.1) $\delta=0.5$ (mid-point rule), $C=1$

$$
\sigma_{t}=4 \quad, \quad \alpha_{u}=0.4627 \quad \text { and } \quad\left\langle\alpha_{g} k\right\rangle=0.2686 \rho \rho^{\prime} u l^{e}
$$

b.2) $\delta=0.5, C=0.5$

$$
\sigma_{t}=8 \quad, \quad \alpha_{u} \simeq 0.125 \quad \text { and } \quad\left\langle\alpha_{g} k\right\rangle=0.4375 \rho c u l^{e}
$$

b.3) $\delta=0.5, C=0.1$

$$
\sigma_{t}=20 \quad, \quad \alpha_{u}=0.05 \quad \text { and } \quad\left\langle\alpha_{g} k\right\rangle=0.475 \rho c u l^{e}
$$

Remark 9. Note that the stabilization parameters evolve from the standard SUPG values $\left(\alpha_{u}=\right.$ 1 and $\left.\left\langle\alpha_{g} k\right\rangle=0\right)$ to the progressive vanishing of the streamline parameter $\left(\alpha_{u} \rightarrow 0\right)$ and $\left\langle\alpha_{g} k\right\rangle \rightarrow 0.5 \rho c u l^{e}$ in the vicinity of a jump for low Courant numbers.

Remark 10. Excellent results have been obtained for all the problems solved in this work using $0.1<C \leq 0.8$.

\subsection{Explicit forward-Euler scheme}

The time discretization of Eq.(84) using an explicit Forward-Euler scheme $(\delta=1)$ gives

$$
\frac{1}{\Delta t} \mathbf{M} \boldsymbol{\phi}^{n+1}+\left[\mathbf{K}-\mathbf{C}+\mathbf{S}-\frac{1}{\Delta t} \mathbf{M}\right] \boldsymbol{\phi}^{n}-\mathbf{f}^{n}=\mathbf{0}
$$

A simple stencil can be found for a mesh of equal length $1 \mathrm{D}$ linear elements using a diagonal form of $\mathbf{M}^{e}$ as

$$
\frac{\rho c l^{e}}{\Delta t} \phi_{i}^{n+1}-\left(\bar{\gamma}+1+\theta-\frac{w}{6}\right) \phi_{i-1}^{n}+2\left(1+\theta+\frac{w}{3}-\frac{\rho c l^{e}}{2 \Delta t}\right) \phi_{i}^{n}-\left(-\bar{\gamma}+1+\theta-\frac{w}{6}\right) \phi_{i+1}^{n}-f_{i}^{n}=0
$$

from which $\phi_{i}^{n+1}$ can be explicitly found.

The values of $\theta$ and $\bar{\gamma}$ in Eq.(99) are computed from Eqs.(22) and (24) respectively with $\alpha_{u}$ and $\alpha_{g}$ obtained as explained in the previous sections.

The stability requirements for the explicit solution require that $[14,69]$

$$
\Delta t \leq \frac{2}{g_{\max }^{e}}
$$

where $g_{\max }^{e}$ is the largest eigenvalue of matrix $\mathbf{H}^{e}$ for all the elements in the mesh with

$$
\mathbf{H}^{e}=\mathbf{K}^{e}-\mathbf{C}^{e}+\mathbf{S}^{e}
$$

\section{EXAMPLES}

\subsection{Steady-state examples solved with equal length 2-noded elements}

The nodal exactness of the steady-state FIC-FEM formulation is shown for a series of convectiondiffusion-radiation problems solved on a coarse uniform mesh of eight 2-noded elements of unit length $\left(l^{e}=1\right)$ in a $1 \mathrm{D}$ domain $\Omega:=[0,8]$. The Dirichlet boundary conditions are $\phi(0)=8$ and $\phi(8)=3$, except for the convection-diffusion-production case where $\phi(0)=\phi(8)=0$ has been taken. Many of these problems were solved in $[17,54]$ using the same mesh.

Figures $2-5$ show the FEM results superimposed on the exact analytical solution for the following problems. 
(a) $\gamma=0, \omega=2$

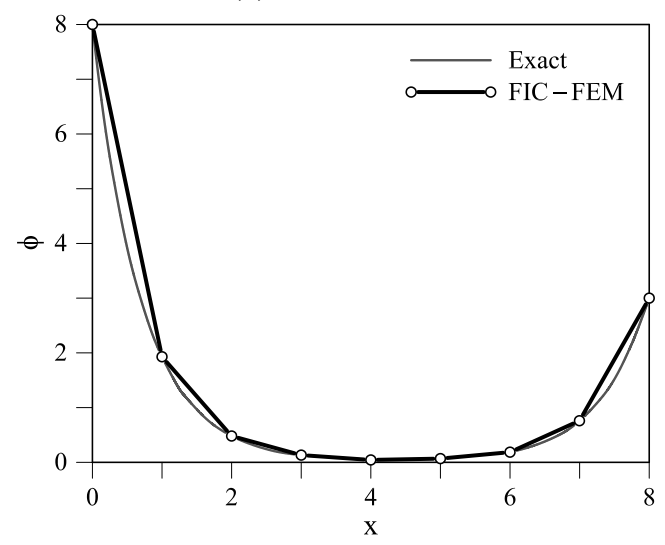

(c) $\gamma=1, \omega=5$

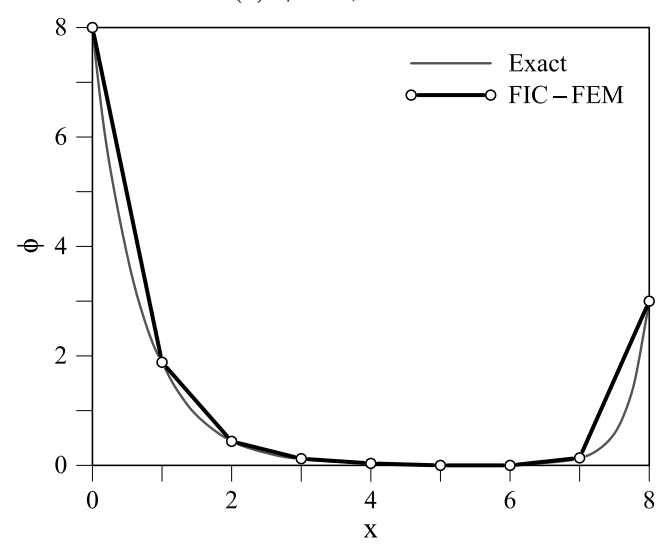

(e) $\gamma=2, \omega=2$

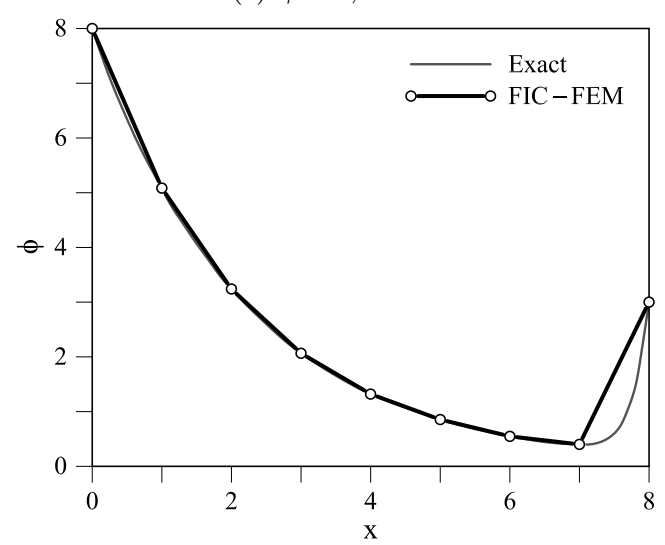

(b) $\gamma=0, \omega=25$

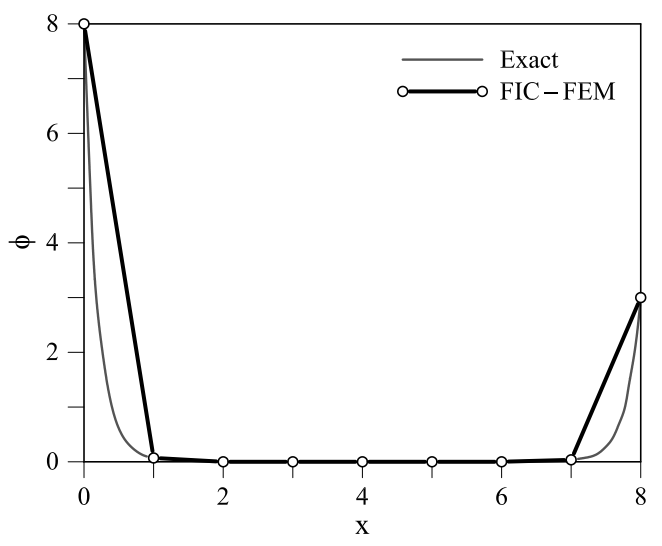

(d) $\gamma=1, \omega=20$

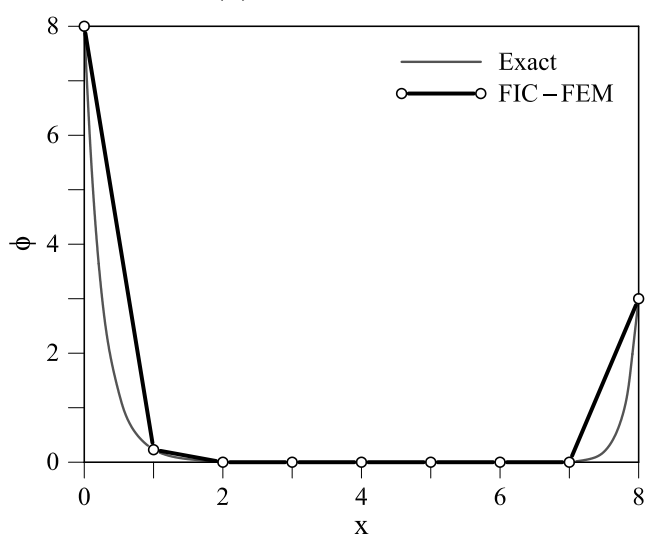

(f) $\gamma=10, \omega=4$

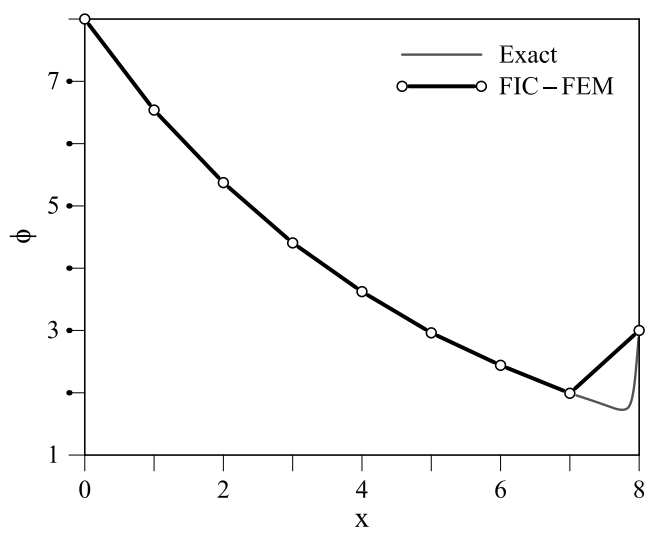

Figure 2: 1D convection-diffusion-absorption problem $(Q=0)$. Exact and FIC-FEM results for a uniform mesh of eight 2-noded elements. (a) $\gamma=0, \omega=2$; (b) $\gamma=0, \omega=25$; (c) $\gamma=1, \omega=5$; (d) $\gamma=1, \omega=20$; (e) $\gamma=2, \omega=2$; (f) $\gamma=10, \omega=4$;

\section{Sourceless case $(Q=0)$}

Convection-diffusion-absorption

- $\gamma=0 \quad, \quad \omega=2($ Figure 2a) 
(a) $\gamma=1, \omega=-20$

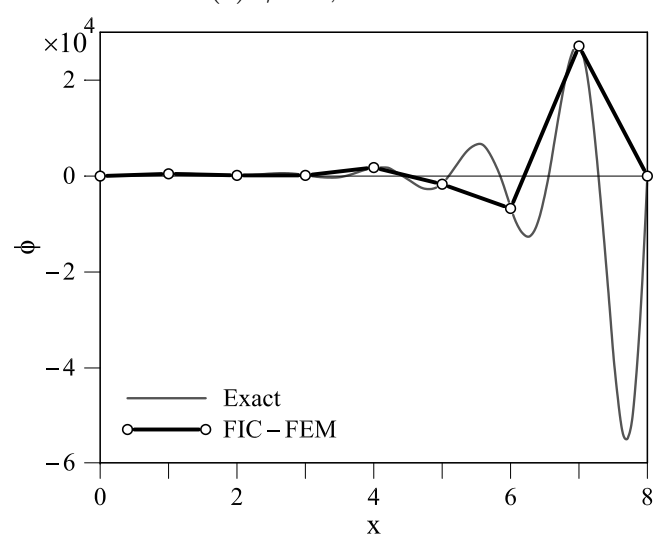

Figure 3: 1D convection-diffusion-production problem $(\gamma=1, \omega=-20, Q=0)$. Exact and FIC-FEM results for a uniform mesh of eight 2-noded elements.

(a) $\gamma=0, \omega=-5$

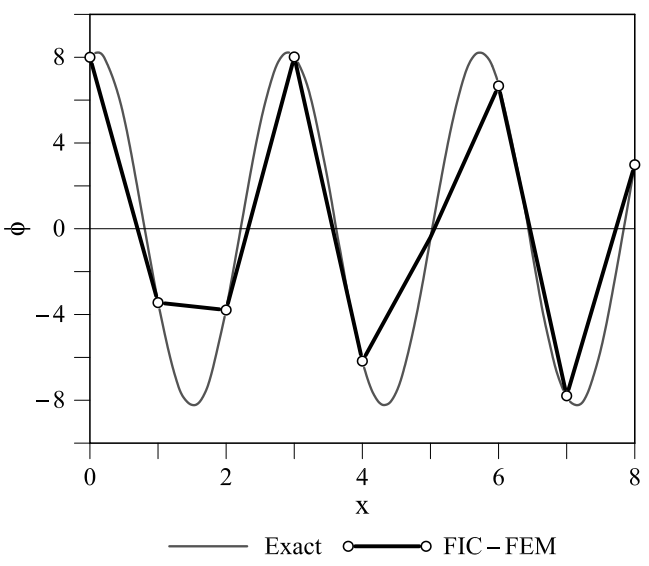

(b) $\gamma=0, \omega=-100$

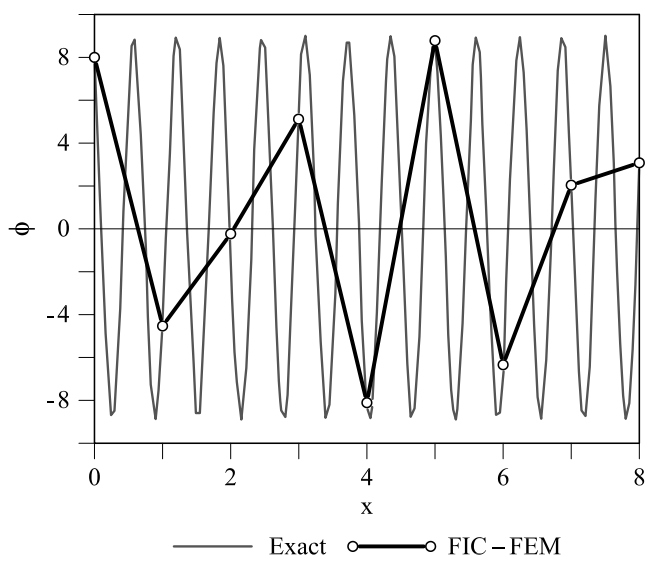

Figure 4: 1D Helmholtz problem $(Q=0)$. Exact and FIC-FEM results for a uniform mesh of eight 2-noded elements. (a) $\gamma=0, \omega=-5$; (b) $\gamma=0, \omega=-100$

- $\gamma=0 \quad, \quad \omega=25$ (Figure $2 \mathrm{~b})$

- $\gamma=1 \quad, \omega=5$ (Figure 2c)

- $\gamma=1 \quad, \quad \omega=20$ (Figure 2d)

- $\gamma=1 \quad, \quad \omega=120$ (Figure 2e)

- $\gamma=2 \quad, \quad \omega=2$ (Figure 2f)

Convection-diffusion-production

- $\gamma=1 \quad, \quad \omega=-20$ (Figure 3)

Helmholtz problem

- $\gamma=0 \quad, \quad \omega=-5$ (Figure 4a)

- $\gamma=0 \quad, \quad \omega=-100$ (Figure 4b) 
(a) $\gamma=2, \omega=2, Q=1$

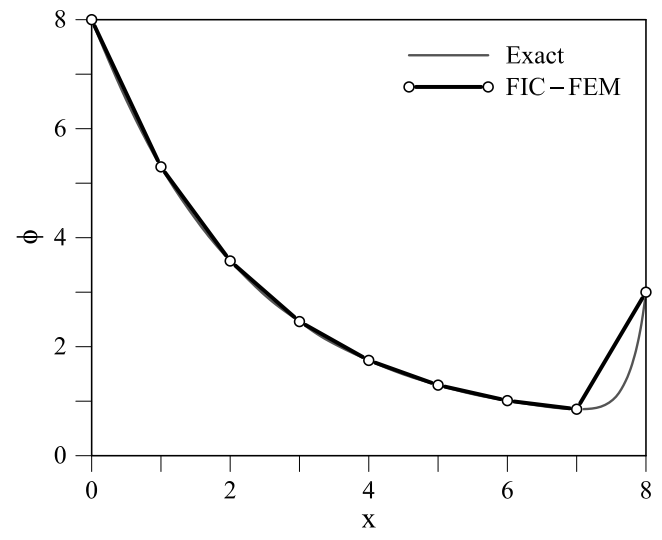

(b) $\gamma=2, \omega=-5, Q=1$

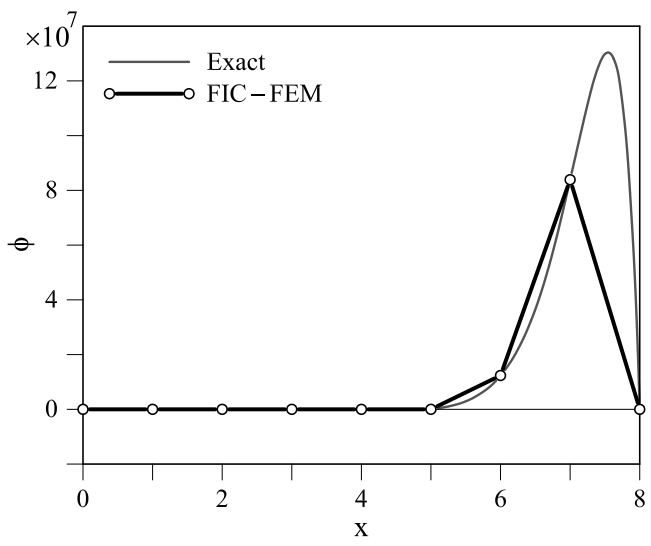

Figure 5: 1D convection-diffusion-production problems. Constant source $(Q=1)$. Exact and FIC-FEM results for a uniform mesh of eight 2-noded elements. (a) $\gamma=2, \omega=2$; (b) $\gamma=2$, $\omega=-5$

\section{Constant source}

- $\gamma=2, \omega=2, Q=1$ (Figure 5a)

- $\gamma=2, \omega=-5, Q=1$ (Figure $5 \mathrm{~b})$

The FIC-FEM solution yields exact nodal solutions for all the problems solved, as expected.

\subsection{Steady-state convection-diffusion-absorption examples solved with elements of different length}

The accuracy of the steady-state FIC-FEM formulation for a non-uniform mesh of eight 2noded linear element has been studied for different problem parameters. The analysis domain $[0,8]$ is discretized into eight elements. The nodal coordinates are shown in Table 1 where the exact and FIC-FEM nodal solutions are given. The numerical and exact results are plotted in Figure 6. The accuracy of the FIC-FEM formulation is remarkable in all cases given the coarseness of the mesh chosen for the simulations. Note the relatively small error of the nodal results in zones where the solution attains very small values (Table 1c) which shows the capability of the FIC-FEM formulation to accurately reproduce the different scales in the solution.

The same non-uniform mesh has been used for solving two problems of diffusion-production (Helmholtz) $(u=0, k=1, s=-1)$ and convective-diffusion-production $(u=1, k=1, s=-2)$. The FIC-FEM and exact solutions are plotted in Figures 7 and 8 . The nodal numerical and exact nodal values for each of the two solutions are listed in Table 2. The accuracy of the FIC-FEM formulation again is remarkable given the oscillatory character of the solutions with values of $\phi$ oscillating from positive to negative in both cases.

Thus, for the Helmholtz problem (Table 2) errors are within $8 \%$ for values of $\left|\phi_{i}\right|>1$ and increase to $\sim 74 \%$ for node 2 where the exact solution approaches to zero.

The same situation occurs for the convective-diffusion-production problem (Table 3) where errors are small $(<10 \%)$ for the higher values of the solution and acceptable for smaller values.

We highlight the coarseness of the mesh used for the purpose of testing the FIC-FEM formulation in a simple irregular mesh situation. Indeed more accurate results can be obtained by simply refining the mesh. 
(a) $u=4, k=1, s=2$

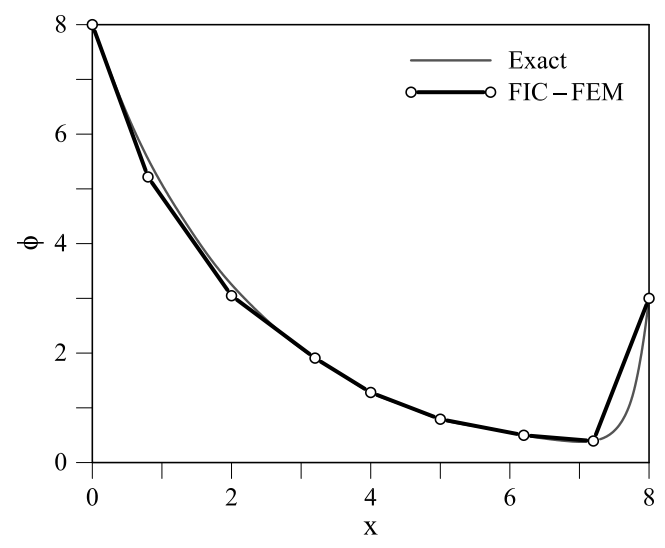

(b) $u=20, k=1, s=1$

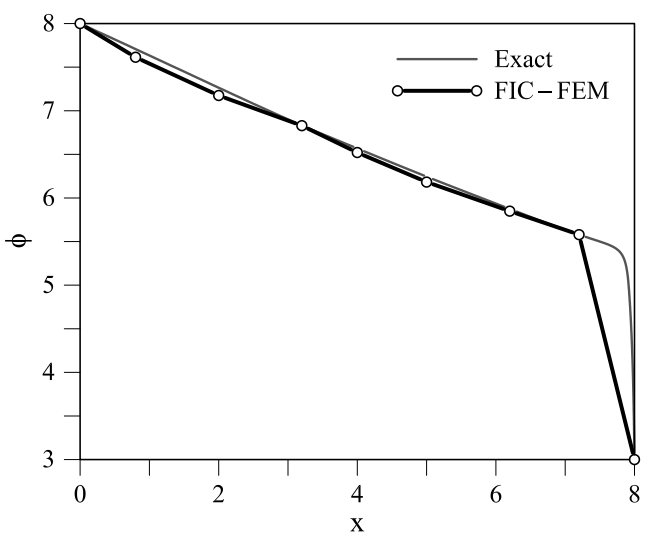

(c) $u=1, k=1, s=20$

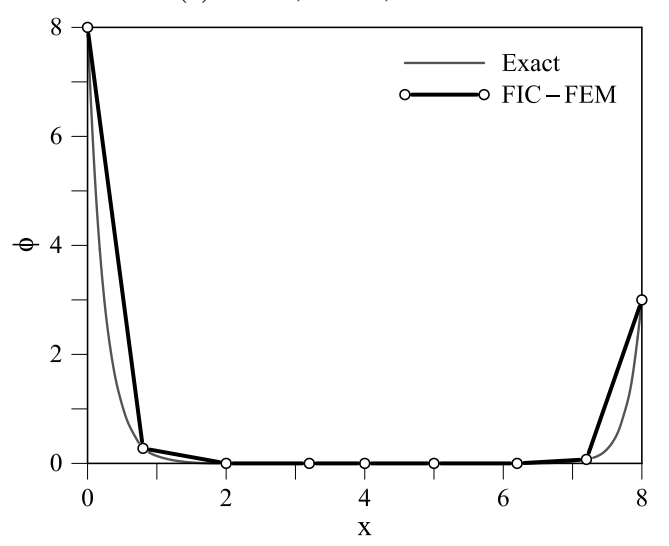

Figure 6: 1D convection-diffusion-absorption problem $(Q=0)$. Exact and FIC-FEM results for an irregular mesh of eight 2-noded elements. (a) $u=4, k=1, s=2$; (b) $u=20, k=1, s=1$; (c) $u=1, k=1, s=20$

\subsection{Performance on layer adapted meshes}

This example illustrates the performance of the proposed FIC-FEM on layer-adapted meshes. We use a Shishkin mesh [63], which is piecewise equidistant and consequently much simpler than several types of layer-adapted meshes in the literature. Given a positive integer $N$, where $N$ is divisible by four, we divide the interval $[0, L]$ into three subintervals

$$
\left[0, \tau_{1} L\right], \quad\left[\tau_{1} L,\left(1-\tau_{2}\right) L\right], \quad \text { and } \quad\left[\left(1-\tau_{2}\right) L, L\right]
$$

Uniform meshes are used on each subinterval with $1+(N / 4)$ nodes in each of $\left[0, \tau_{1} L\right]$ and $[(1-$ $\left.\left.\tau_{2}\right) L, L\right]$, and $1+(N / 2)$ nodes in $\left[\tau_{1} L,\left(1-\tau_{2}\right) L\right]$. The mesh-transition parameters $\tau_{1}$ and $\tau_{2}$ are defined as

$$
\begin{gathered}
\tau_{1}=\min \left\{\frac{1}{4}, \frac{2}{\left|\mu_{1}\right|} \ln N\right\}, \quad \tau_{2}=\min \left\{\frac{1}{4}, \frac{2}{\left|\mu_{2}\right|} \ln N\right\} \\
\mu_{1}=\frac{u L}{2 k}-\sqrt{\left(\frac{u L}{2 k}\right)^{2}+\frac{s L^{2}}{k}}, \quad \mu_{2}=\frac{u L}{2 k}+\sqrt{\left(\frac{u L}{2 k}\right)^{2}+\frac{s L^{2}}{k}}
\end{gathered}
$$

The layer-strength parameters $\mu_{1}$ and $\mu_{2}$ appear in the fundamental solutions, viz. $\exp \left(\mu_{1} x / L\right)$ and $\exp \left(\mu_{2} x / L\right)$ of Eq. (4). Set $i_{0}=N / 4$. The transition points of the Shishkin mesh $X_{\mathrm{s}}^{N}$ are 
(a) $u=4, k=1, s=2$

\begin{tabular}{|c|c|c|c|c|}
\hline Node & Coordinate & Exact & FIC-FEM & \% error \\
\hline 1 & 0 & 8,0000 & 8,0000 & 0 \\
2 & 0,8 & 5,5837 & 5,2336 & 6,27 \\
3 & 2 & 3,2559 & 3,0523 & 6,25 \\
4 & 3,2 & 1,8985 & 1,8954 & 0,16 \\
5 & 4 & 1,3251 & 1,2831 & 3,16 \\
6 & 5 & 0,8454 & 0,7920 & 6,31 \\
7 & 6,2 & 0,4939 & 0,4789 & 3,03 \\
8 & 7,2 & 0,3936 & 0,3932 & 0,10 \\
9 & 8,0 & 3,0000 & 3,0000 & 0 \\
\hline
\end{tabular}

(b) $u=20, k=1, s=1$

\begin{tabular}{|c|c|c|c|c|}
\hline Node & Coordinate & Exact & FIC-FEM & \% error \\
\hline 1 & 0 & 8,0000 & 8,0000 & 0 \\
2 & 0,8 & 7,6871 & 7,6126 & 0,97 \\
3 & 2 & 7,2405 & 7,1704 & 0,97 \\
4 & 3,2 & 6,8199 & 6,8199 & 0 \\
5 & 4 & 6,5531 & 6,5212 & 0,48 \\
6 & 5 & 6,2343 & 6,1739 & 0,97 \\
7 & 6,2 & 5,8721 & 5,8435 & 0,48 \\
8 & 7,2 & 5,5864 & 5,5864 & 0 \\
9 & 8,0 & 3,0000 & 3,0000 & 0 \\
\hline
\end{tabular}

(c) $u=1, k=1, s=20$

\begin{tabular}{|c|c|c|c|c|}
\hline Node & Coordinate & Exact & FIC-FEM & $\%$ error \\
\hline 1 & 0 & 8,0000 & 8,0000 & 0 \\
2 & 0,8 & 0,3261 & 0,2670 & 18,12 \\
3 & 2 & 0,2684 E-02 & 0,2197 E-02 & 18,14 \\
4 & 3,2 & 0,2209 E-04 & 0,2140 E -04 & 3,12 \\
5 & 4 & 0,9065 E-06 & 0,7333 E-06 & 19,10 \\
6 & 5 & 0,9342 E-06 & 0,8419 E-06 & 9,88 \\
7 & 6,2 & 0,3702 E-03 & 0,3092 E-03 & 16,47 \\
8 & 7,2 & 0,5495 E-01 & 0,4997 E-01 & 9,06 \\
9 & 8,0 & 3,0000 & 3,0000 & 0 \\
\hline
\end{tabular}

Table 1: 1D convection-diffusion-production problems $(Q=0)$. Exact and FIC-FEM nodal results for an irregular mesh of eight 2-noded elements (results are plotted in Figure 6)

$$
\begin{aligned}
& x_{i_{0}}=\tau_{1} L \text { and } x_{N-i_{0}}=\left(1-\tau_{2}\right) L \\
& X_{\mathrm{s}}^{N}: 0=x_{0}<x_{1}<\ldots<x_{i_{0}}<\ldots<x_{N-i_{0}}<\ldots<x_{N}=L
\end{aligned}
$$

We use the double mesh method [12] to compute the experimental rates of convergence. To compute the double mesh error we need each node of the coarse mesh $X_{\mathrm{s}}^{N}$ to coincide with a unique node in the fine mesh $X_{\mathrm{S}}^{2 N}$. Usually this will not be the case for Shishkin meshes as the mesh partition has a nonlinear dependence on $N$. In order to compute the double mesh error, we compute not only $\phi^{N}$ (solution computed using $X_{\mathrm{s}}^{N}$ ), but also another approximate solution $\widetilde{\phi}^{N}$ computed on a modified Shishkin mesh $\widetilde{X}_{s}^{N}[64]$. The modified Shishkin mesh $\widetilde{X}_{\mathrm{s}}^{N}$ is constructed using the slightly altered mesh-transition parameters

$$
\widetilde{\tau}_{1}=\min \left\{\frac{1}{4}, \frac{2}{\left|\mu_{1}\right|} \ln \frac{N}{2}\right\}, \quad \widetilde{\tau}_{2}=\min \left\{\frac{1}{4}, \frac{2}{\left|\mu_{2}\right|} \ln \frac{N}{2}\right\}
$$




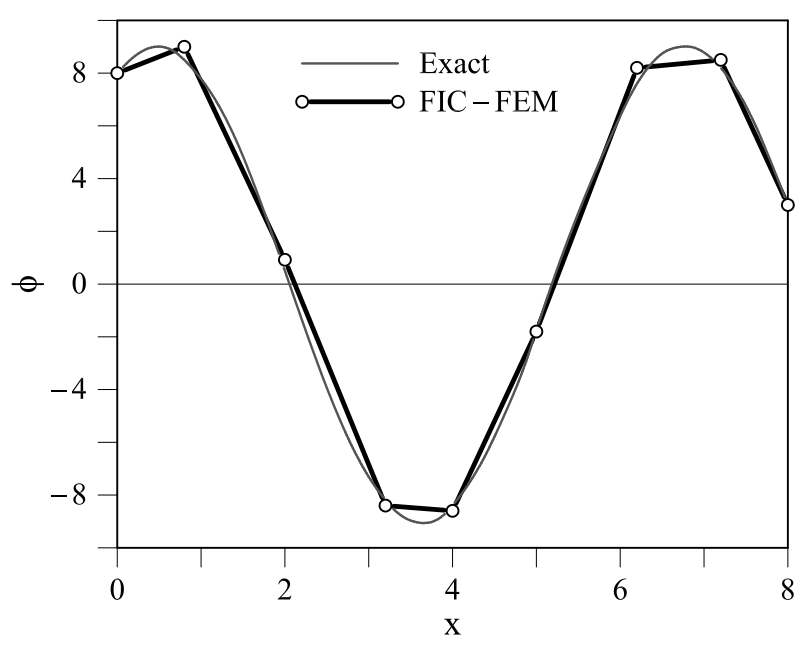

Figure 7: 1D Helmholtz problem $(u=0, k=1, s=-1, Q=0)$. Exact and FIC-FEM results for an irregular mesh of eight 2-noded element

(a) $u=0, k=1, s=-1$

\begin{tabular}{|c|c|c|c|c|}
\hline Node & Coordinate & Exact & FIC-FEM & \% error \\
\hline 1 & 0 & 8,0000 & 8,0000 & 0 \\
2 & 0,8 & 8,5829 & 9,0211 & 4,98 \\
3 & 2 & 0,4979 &, 8652 & 73,77 \\
4 & 3,2 & $-8,2320$ & $-8,3941$ & 1,97 \\
5 & 4 & $-8,4144$ & $-8,6356$ & 2,63 \\
6 & 5 & $-1,7666$ & 1,7829 & 0,92 \\
7 & 6,2 & 7,6226 & 8,2224 & 7,87 \\
8 & 7,2 & 8,2072 & 8,5626 & 4,33 \\
9 & 8,0 & 3,0000 & 3,0000 & 0 \\
\hline
\end{tabular}

Table 2: 1D problem $(u=0, k=1, s=-1, Q=0)$. Exact and FIC-FEM results for an irregular mesh of eight 2-noded element (results are plotted in Figure 7)

(a) $u=1, k=1, s=-2$

\begin{tabular}{|c|c|c|c|c|}
\hline Node & Coordinate & Exact & FIC-FEM & $\%$ error \\
\hline 1 & 0 & 8,0000 & 8,0000 & 0 \\
2 & 0,8 & 1,2217 & 1,6667 & 36,42 \\
3 & 2 & $-23,7333$ & $-27,7740$ & 17,03 \\
4 & 3,2 & $-2,6157$ & $-3,8480$ & 47,11 \\
5 & 4 & 54,3764 & 52,5686 & 3,32 \\
6 & 5 & 78,0550 & 80,7289 & 3,43 \\
7 & 6,2 & $-134,8567$ & $-147,3987$ & 9,3 \\
8 & 7,2 & $-278,3221$ & $-279,2699$ & 0,34 \\
9 & 8,0 & 3,0000 & 3,0000 & 0 \\
\hline
\end{tabular}

Table 3: 1D convection-diffusion-production problem $(u=1, k=1, s=-2, Q=0)$. Exact and FIC-FEM nodal results for an irregular mesh of eight 2-noded elements (results are plotted in Figure 8) 


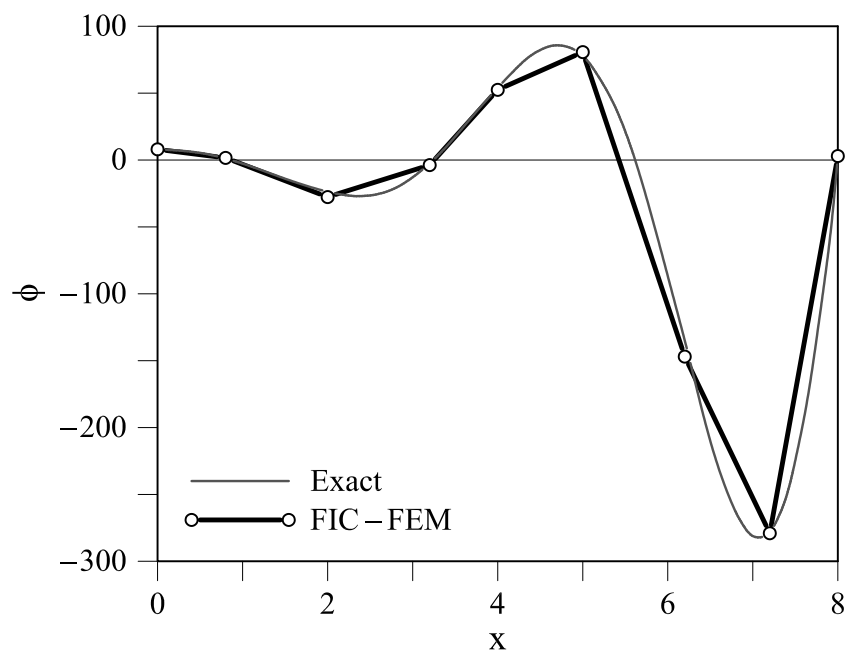

Figure 8: 1D convection-diffusion-production problem $(u=1, k=1, s=-2, Q=0)$. Exact and FIC-FEM results for an irregular mesh of eight 2-noded element

Then for $i=0,1, \ldots, N$, the $i$ th node of the mesh $X_{\mathrm{s}}^{N}$ coincides with the $(2 i)$ th node of the mesh $\widetilde{X}_{\mathrm{s}}^{2 N}$. We set $L=8, u=5, s=20, f=0$ and the boundary conditions $\phi_{0}^{N}=\widetilde{\phi}_{0}^{N}=8$ and $\phi_{N}^{N}=\widetilde{\phi}_{N}^{N}=3$. We generate a sequence of Shishkin meshes using $N=2^{m}, m=\{7,8, \ldots, 12\}$ and solve the problem for a sequence of diffusion coefficients $k=0.25^{n}, n=\{1,2, \ldots, 10\}$. For each $N$ and $k$, we compute the double mesh error $\widetilde{E}_{k}^{N}$ as

$$
\widetilde{E}_{k}^{N}=\max _{0 \leq i \leq N}\left|\phi_{i}^{N}-\widetilde{\phi}_{2 i}^{2 N}\right|
$$

For the stabilized FIC-FEM we assume a convergence of order $N^{-r}$ for some $r$ (a natural expectation from a stabilized method) and compute the convergence rate $r$ for each fixed $k$ from

$$
R_{k}^{N}=\frac{\ln \widetilde{E}_{k}^{N}-\ln \widetilde{E}_{k}^{2 N}}{\ln 2}
$$

Table 4 shows that the proposed FIC-FEM has an estimated $k$-uniform convergence of order $N^{-2}$. It suggests that the stabilized FIC-FEM may satisfy the following error estimate for Shishkin meshes.

$$
\max _{0 \leq i \leq N}\left|\phi\left(x_{i}\right)-\phi_{i}^{N}\right| \leq C N^{-2}
$$

It is well known [21] that the Galerkin-FEM with linear finite elements statisfies the following error estimate for Shishkin meshes.

$$
\max _{0 \leq i \leq N}\left|\phi\left(x_{i}\right)-\phi_{i}^{N}\right| \leq C\left(N^{-1} \ln N\right)^{2}
$$

To verify the above error estimate we compute the convergence rate for each fixed $k$ from

$$
R_{k}^{N}=\frac{\ln \widetilde{E}_{k}^{N}-\ln \widetilde{E}_{k}^{2 N}}{\ln \left(\frac{2 \ln N}{\ln 2 N}\right)}=\frac{\ln \widetilde{E}_{k}^{N}-\ln \widetilde{E}_{k}^{2 N}}{\ln \left(\frac{2 m}{m+1}\right)}, \quad \text { for } \quad N=2^{m} \text { and } m=7,8, \ldots, 12 .
$$

Table 5 shows as expected that the Galerkin-FEM with linear finite elements has an estimated $k$-uniform convergence of order $\left(N^{-1} \ln N\right)^{2}$.

In Figure 9, we choose $k=0.25$ and illustrate the FIC-FEM solution computed on a Shishkin mesh with $N=2^{5}$ nodes. The Shishkin mesh transition points $x_{i_{0}}=2$ and $x_{N-i_{0}}=7.704$ are also shown. 


\begin{tabular}{ccccccc}
\hline$k$ & $N=128$ & 256 & 512 & 1024 & 2048 & 4096 \\
\hline $2.5000 \mathrm{e}-1$ & $1.9612 \mathrm{e}-5$ & $4.8248 \mathrm{e}-6$ & $1.1606 \mathrm{e}-6$ & $2.7720 \mathrm{e}-7$ & $6.5999 \mathrm{e}-8$ & $1.5678 \mathrm{e}-8$ \\
& 2.0232 & 2.0556 & 2.0658 & 2.0704 & 2.0737 & \\
$6.2500 \mathrm{e}-2$ & $1.3838 \mathrm{e}-5$ & $3.6689 \mathrm{e}-6$ & $9.2874 \mathrm{e}-7$ & $2.3119 \mathrm{e}-7$ & $5.7273 \mathrm{e}-8$ & $1.4170 \mathrm{e}-8$ \\
& 1.9153 & 1.982 & 2.0062 & 2.0131 & 2.015 & \\
$1.5625 \mathrm{e}-2$ & $1.3193 \mathrm{e}-5$ & $3.2052 \mathrm{e}-6$ & $8.3014 \mathrm{e}-7$ & $2.1045 \mathrm{e}-7$ & $5.2765 \mathrm{e}-8$ & $1.3176 \mathrm{e}-8$ \\
& 2.0412 & 1.949 & 1.9799 & 1.9958 & 2.0016 & \\
$3.9063 \mathrm{e}-3$ & $1.3211 \mathrm{e}-5$ & $3.0802 \mathrm{e}-6$ & $7.9945 \mathrm{e}-7$ & $2.0349 \mathrm{e}-7$ & $5.1313 \mathrm{e}-8$ & $1.2872 \mathrm{e}-8$ \\
& 2.1006 & 1.946 & 1.974 & 1.9876 & 1.9951 & \\
$9.7656 \mathrm{e}-4$ & $1.3215 \mathrm{e}-5$ & $3.0488 \mathrm{e}-6$ & $7.9176 \mathrm{e}-7$ & $2.0164 \mathrm{e}-7$ & $5.0868 \mathrm{e}-8$ & $1.2774 \mathrm{e}-8$ \\
& 2.1158 & 1.9451 & 1.9733 & 1.9869 & 1.9936 & \\
$2.4414 \mathrm{e}-4$ & $1.3216 \mathrm{e}-5$ & $3.0410 \mathrm{e}-6$ & $7.8984 \mathrm{e}-7$ & $2.0118 \mathrm{e}-7$ & $5.0758 \mathrm{e}-8$ & $1.2747 \mathrm{e}-8$ \\
& 2.1196 & 1.9449 & 1.9731 & 1.9867 & 1.9934 & \\
$6.1035 \mathrm{e}-5$ & $1.3216 \mathrm{e}-5$ & $3.0390 \mathrm{e}-6$ & $7.8936 \mathrm{e}-7$ & $2.0106 \mathrm{e}-7$ & $5.0730 \mathrm{e}-8$ & $1.2741 \mathrm{e}-8$ \\
& 2.1206 & 1.9449 & 1.9731 & 1.9867 & 1.9934 & \\
$1.5259 \mathrm{e}-5$ & $1.3216 \mathrm{e}-5$ & $3.0385 \mathrm{e}-6$ & $7.8924 \mathrm{e}-7$ & $2.0103 \mathrm{e}-7$ & $5.0723 \mathrm{e}-8$ & $1.2739 \mathrm{e}-8$ \\
& 2.1208 & 1.9448 & 1.973 & 1.9867 & 1.9934 & \\
$3.8147 \mathrm{e}-6$ & $1.3216 \mathrm{e}-5$ & $3.0384 \mathrm{e}-6$ & $7.8921 \mathrm{e}-7$ & $2.0102 \mathrm{e}-7$ & $5.0722 \mathrm{e}-8$ & $1.2739 \mathrm{e}-8$ \\
& 2.1209 & 1.9448 & 1.973 & 1.9867 & 1.9934 & \\
$9.5367 \mathrm{e}-7$ & $1.3216 \mathrm{e}-5$ & $3.0384 \mathrm{e}-6$ & $7.8920 \mathrm{e}-7$ & $2.0102 \mathrm{e}-7$ & $5.0721 \mathrm{e}-8$ & $1.2739 \mathrm{e}-8$ \\
& 2.1209 & 1.9448 & 1.973 & 1.9867 & 1.9934 & \\
\hline
\end{tabular}

Table 4: Errors $\widetilde{E}_{k}^{N}$ and convergence rates $R_{k}^{N}=\left(\ln \widetilde{E}_{k}^{N}-\ln \widetilde{E}_{k}^{2 N}\right) / \ln 2$ for the FIC-FEM method generated using $L=8, u=5, s=20, f=0, k=0.25^{n}, n=\{1,2, \ldots, 10\}, N=2^{m}, m=$ $\{7,8, \ldots, 12\}$ and boundary conditions $\phi_{0}^{N}=\widetilde{\phi}_{0}^{N}=8$ and $\phi_{N}^{N}=\widetilde{\phi}_{N}^{N}=3$. 


\begin{tabular}{ccccccc}
\hline$k$ & N $=128$ & 256 & 512 & 1024 & 2048 & 4096 \\
\hline $2.5000 \mathrm{e}-1$ & $5.5786 \mathrm{e}-3$ & $1.8129 \mathrm{e}-3$ & $5.7243 \mathrm{e}-4$ & $1.7653 \mathrm{e}-4$ & $5.3399 \mathrm{e}-5$ & $1.5887 \mathrm{e}-5$ \\
& 2.0085 & 2.0036 & 2.0014 & 2 & 2 & \\
$6.2500 \mathrm{e}-2$ & $6.1182 \mathrm{e}-3$ & $1.9873 \mathrm{e}-3$ & $6.2741 \mathrm{e}-4$ & $1.9347 \mathrm{e}-4$ & $5.8524 \mathrm{e}-5$ & $1.7412 \mathrm{e}-5$ \\
& 2.0094 & 2.0039 & 2.0015 & 2.0001 & 2 & \\
$1.5625 \mathrm{e}-2$ & $6.3215 \mathrm{e}-3$ & $2.0530 \mathrm{e}-3$ & $6.4810 \mathrm{e}-4$ & $1.9985 \mathrm{e}-4$ & $6.0453 \mathrm{e}-5$ & $1.7985 \mathrm{e}-5$ \\
& 2.0097 & 2.004 & 2.0015 & 2.0001 & 2.0001 & \\
$3.9063 \mathrm{e}-3$ & $6.3793 \mathrm{e}-3$ & $2.0716 \mathrm{e}-3$ & $6.5398 \mathrm{e}-4$ & $2.0166 \mathrm{e}-4$ & $6.1001 \mathrm{e}-5$ & $1.8148 \mathrm{e}-5$ \\
& 2.0098 & 2.004 & 2.0015 & 2.0001 & 2.0001 & \\
$9.7656 \mathrm{e}-4$ & $6.3942 \mathrm{e}-3$ & $2.0764 \mathrm{e}-3$ & $6.5550 \mathrm{e}-4$ & $2.0213 \mathrm{e}-4$ & $6.1142 \mathrm{e}-5$ & $1.8191 \mathrm{e}-5$ \\
& 2.0098 & 2.004 & 2.0015 & 2.0001 & 2.0001 & \\
$2.4414 \mathrm{e}-4$ & $6.3980 \mathrm{e}-3$ & $2.0777 \mathrm{e}-3$ & $6.5588 \mathrm{e}-4$ & $2.0225 \mathrm{e}-4$ & $6.1178 \mathrm{e}-5$ & $1.8201 \mathrm{e}-5$ \\
& 2.0098 & 2.004 & 2.0015 & 2.0001 & 2.0001 & \\
$6.1035 \mathrm{e}-5$ & $6.3989 \mathrm{e}-3$ & $2.0780 \mathrm{e}-3$ & $6.5598 \mathrm{e}-4$ & $2.0228 \mathrm{e}-4$ & $6.1187 \mathrm{e}-5$ & $1.8204 \mathrm{e}-5$ \\
& 2.0098 & 2.004 & 2.0015 & 2.0001 & 2.0001 & \\
$1.5259 \mathrm{e}-5$ & $6.3992 \mathrm{e}-3$ & $2.0780 \mathrm{e}-3$ & $6.5600 \mathrm{e}-4$ & $2.0229 \mathrm{e}-4$ & $6.1189 \mathrm{e}-5$ & $1.8205 \mathrm{e}-5$ \\
& 2.0098 & 2.004 & 2.0015 & 2.0001 & 2.0001 & \\
$3.8147 \mathrm{e}-6$ & $6.3992 \mathrm{e}-3$ & $2.0781 \mathrm{e}-3$ & $6.5601 \mathrm{e}-4$ & $2.0229 \mathrm{e}-4$ & $6.1190 \mathrm{e}-5$ & $1.8205 \mathrm{e}-5$ \\
& 2.0098 & 2.004 & 2.0015 & 2.0001 & 2.0001 & \\
$9.5367 \mathrm{e}-7$ & $6.3992 \mathrm{e}-3$ & $2.0781 \mathrm{e}-3$ & $6.5601 \mathrm{e}-4$ & $2.0229 \mathrm{e}-4$ & $6.1190 \mathrm{e}-5$ & $1.8205 \mathrm{e}-5$ \\
& 2.0098 & 2.004 & 2.0015 & 2.0001 & 2.0001 & \\
\hline
\end{tabular}

Table 5: Errors $\widetilde{E}_{k}^{N}$ and convergence rates $R_{k}^{N}=\left(\ln \widetilde{E}_{k}^{N}-\ln \widetilde{E}_{k}^{2 N}\right) / \ln \left(\frac{2 \ln N}{\ln 2 N}\right)$ for the GalerkinFEM method generated using $L=8, u=5, s=20, f=0, k=0.25^{n}, n=\{1,2, \ldots, 10\}, N=$ $2^{m}, m=\{7,8, \ldots, 12\}$ and boundary conditions $\phi_{0}^{N}=\widetilde{\phi}_{0}^{N}=8$ and $\phi_{N}^{N}=\widetilde{\phi}_{N}^{N}=3$. 


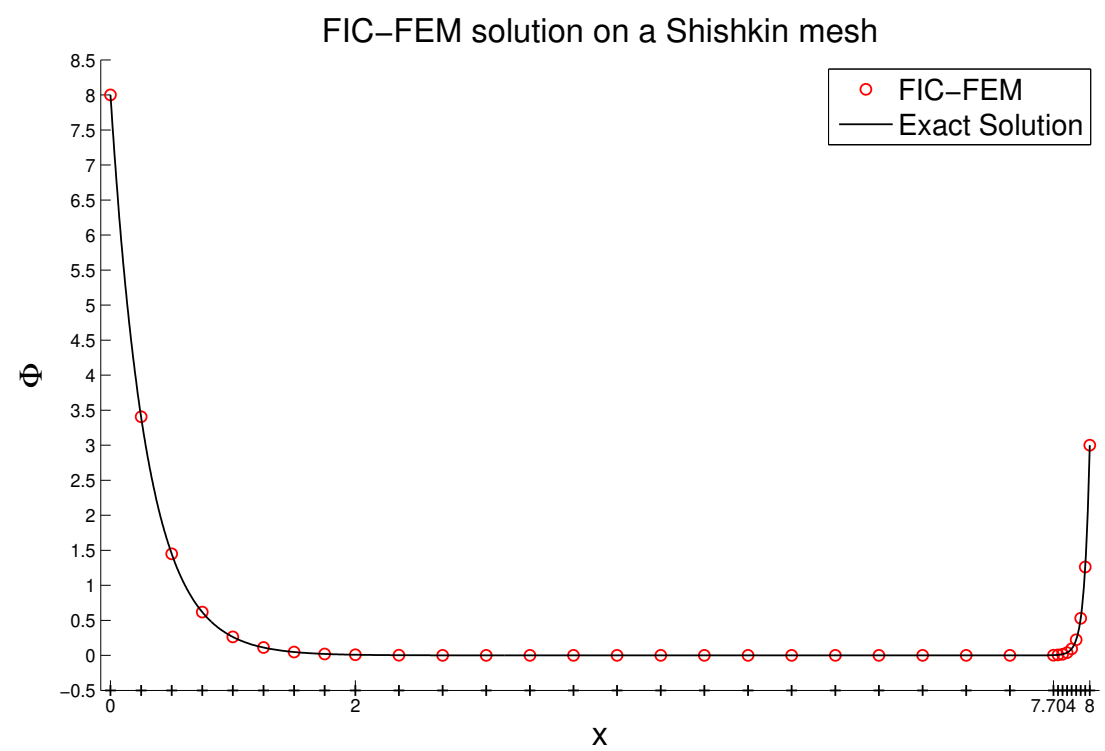

Figure 9: The FIC-FEM solution computed on a Shishkin mesh using $L=8, u=5, s=20, f=$ $0, k=0.25, N=2^{5}$ and boundary conditions $\phi_{0}^{N}=8$ and $\phi_{N}^{N}=3$. The mesh transition points $x_{i_{0}}=2$ and $x_{N-i_{0}}=7.704$ are also shown.

\subsection{Transient convection-diffusion-absorption problem solved with equal length ele- ments}

The analysis domain $x \in[0,8]$ is discretised into eight 2 -node elements of equal length. The convection, diffusion and reaction coefficients are chosen as $u=8, k=2$ and $s=2$. The density $\rho$ and the specific flux $c$ are chosen such that $\rho c=1$. The problem data yields the dimensionless numbers $\gamma=2$ and $\omega=1$. The Dirichlet boundary conditions $\phi(x=0)=3$ and $\phi(x=8)=8$ are employed. The initial solution is chosen to have a linear profile. The transient solution was obtained iteratively as explained in Section 8.2 using the implicit midpoint rule as the time integrator $(\delta=0.5)$ and considering a time step of $\Delta t=0.0625 \mathrm{~s}$. This corresponds to a CFL number $C=0.5$. Figure 10 shows the solutions obtained by the Galerkin-FEM (Fig. 10a), the SUPG method (Fig. 10b) and two variants of the FIC-FEM method (Figs. 10c and 10d). In the first version of the FIC-FEM method the stabilization parameters were taken as those derived for the steady-state problem. In the second version of the FIC-FEM method the stabilization parameters obtained by including a dispersion control model (i.e. a pseudo Damköler number $\left.\sigma_{t}\right)$ is considered. An exponential layer gradually develops at the right boundary which triggers a global instability in the Galerkin FEM. This global instability is successfully controlled by the SUPG (well-known result) and the FIC-FEM method without a dispersion control model. As there is no significant dispersive phenomenon occurring in this problem, the FIC-FEM method with a dispersion control model yields a similar solution.

\subsection{Transient pure convective transport problem solved with equal length elements}

The pure convective transport problem is studied here, i.e. the coefficients $k=s=f=0$ and $u=1$. The $1 \mathrm{D}$ domain is taken as $x \in[0,1]$ and the Dirichlet boundary condition $\phi(x=0)=0$ is employed. The initial solution is a double rectangular pulse with simple discontinuities.

$$
\phi(x, t=0)=\left\{\begin{array}{ccc}
1 & \forall & x \in[0.1,0.2] \cup[0.3,0.4] \\
0 & \text { else }
\end{array}\right.
$$

The amplitude spectrum of this initial solution is rich in high wave numbers. It is a challenging 
(a)

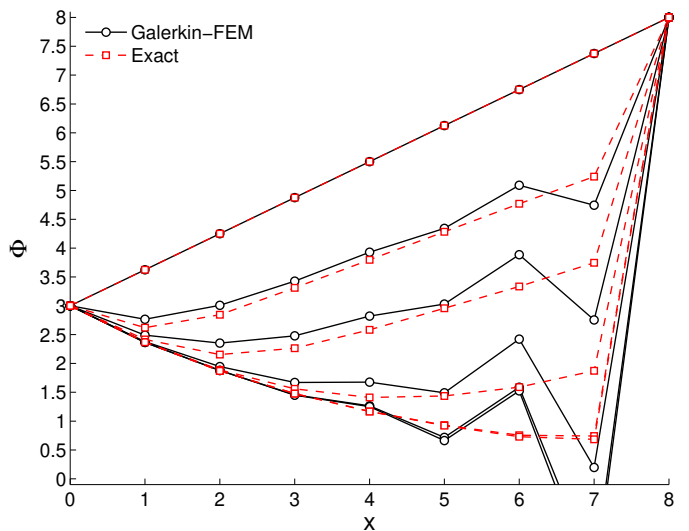

(c)

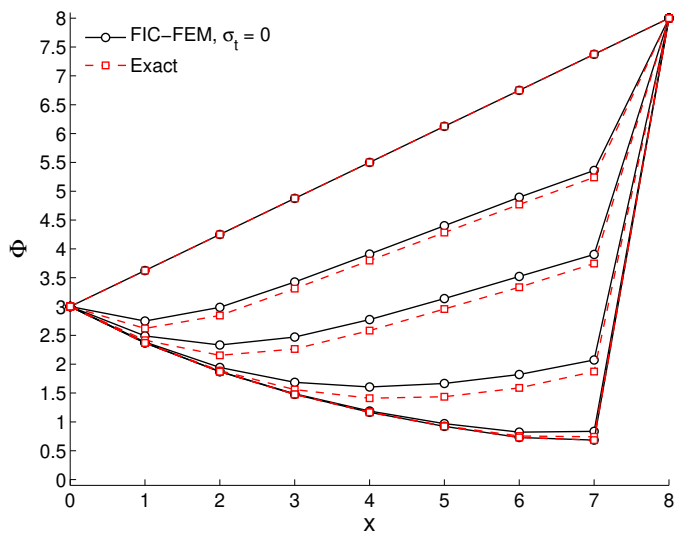

(b)

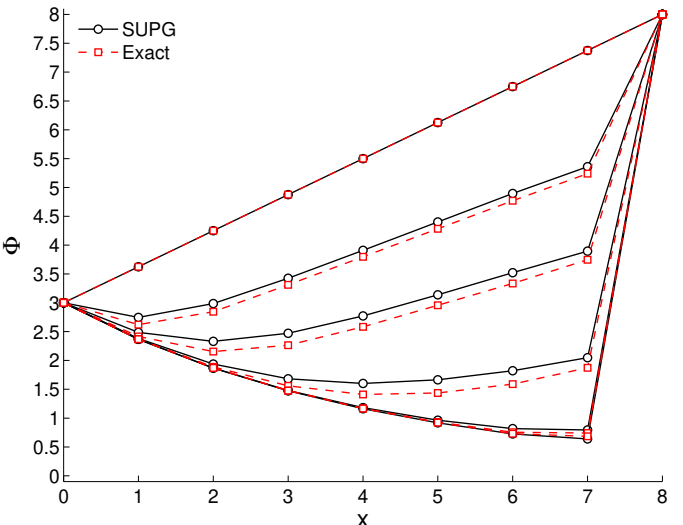

(d)

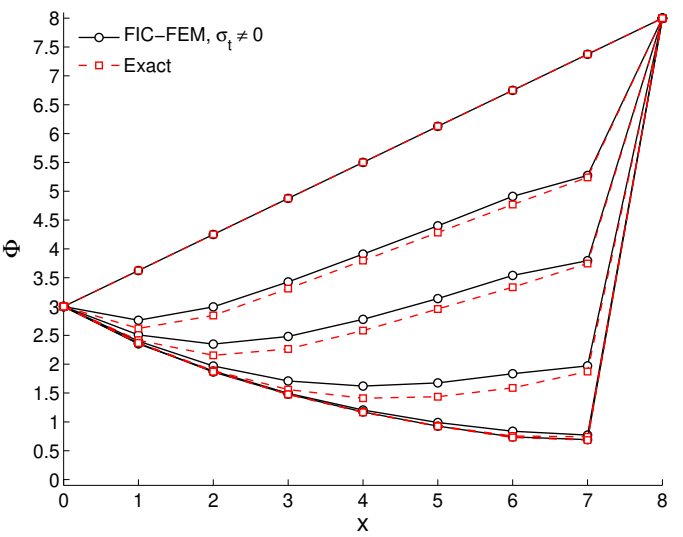

Figure 10: Transient 1D convection-diffusion-reaction problem with $\mathrm{Q}=0$. Exact solution is compared with the Gakerkin, SUPG and FIC-FEM solutions for a uniform mesh of eight 2-noded elements. The solutions are plotted at time $0.125 \mathrm{~s}, 0.25 \mathrm{~s}, 0.5 \mathrm{~s}, 1 \mathrm{~s}$ and $2 \mathrm{~s}$.

problem to validate the control of dispersive oscillations and temporal accuracy. The domain is discretized with 2002 -node elements. Two time steps $\Delta t=0.001 \mathrm{~s}$ and $\Delta t=0025$ were considered, which corresponds to CFL numbers $C=0.2$ and $C=0.5$, respectively. The linearisation was done using a fixed-point method and a tolerance of $10^{-4}$ for the relative error in the Euclidean vector norm was used. For the pure convection problem, the FIC-FEM method without the dispersion control model (letting $\sigma_{t}=0$ ) reduces to the SUPG method. It is well-known that the SUPG method is not immune to dispersive errors $[39,40]$. Appreciable control over the dispersive oscillations is obtained (see Figure 11) with the FIC-FEM method with $\sigma_{t} \neq 0$ and given by Eq. $(90)$.

\section{CONCLUDING REMARKS}

We have presented a stabilized FIC-FEM formulation for 1D advection-diffusion-reaction problems involving two stabilization parameters. The expression of these parameters ensuring an accurate (and many times exact) nodal solution has been obtained for the steady-state and transient cases for a range of physical parameters using linear 2-noded elements. The resulting stabilized formulation will be useful for the accurate solution of many practical problems in engineering and applied sciences involving the $1 \mathrm{D}$ advection-diffusion-reaction equation.

The solution of multidimensional convection-diffusion-reaction problems can develop exponen- 
(a) $\Delta t=0.001 \mathrm{~s}$

FIC, Time $=0 \mathrm{~s}, 0.1,0.5 \mathrm{~s}$; SUPG Initialization

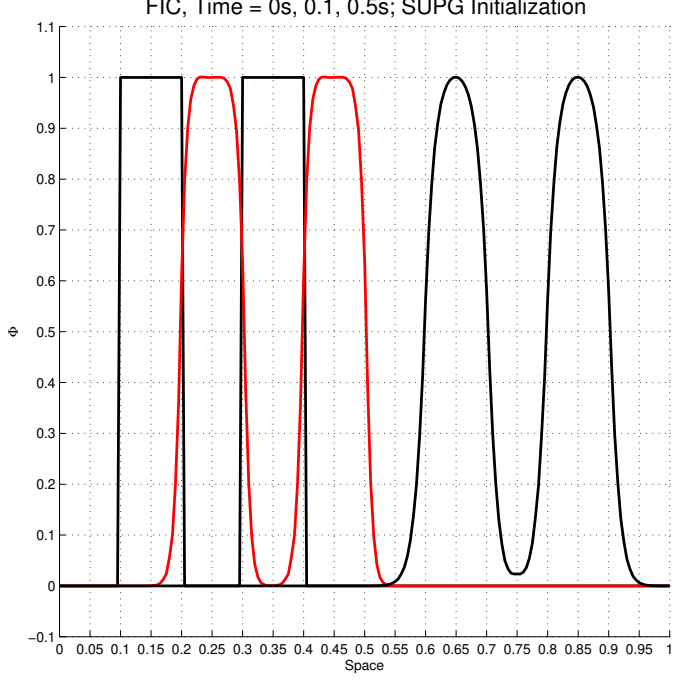

(c) $\Delta t=0.001 \mathrm{~s}$

FIC, Time $=0-0.5 \mathrm{~s} ;$ SUPG Initialization

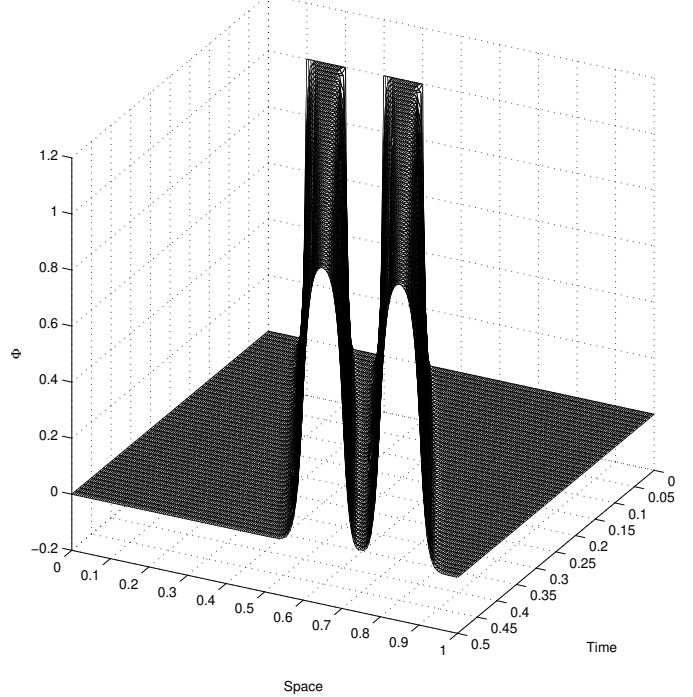

(b) $\Delta t=0.0025 \mathrm{~s}$

FIC, Time $=0 \mathrm{~s}, 0.1,0.5 \mathrm{~s} ;$ SUPG Initialization

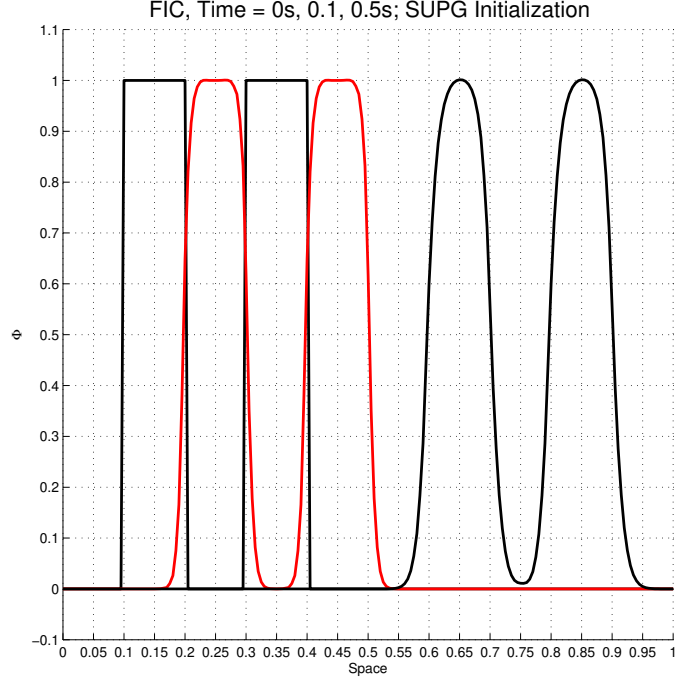

(d) $\Delta t=0.0025 \mathrm{~s}$

$\mathrm{FIC}$, Time $=0-0.5 \mathrm{~s} ;$ SUPG Initialization

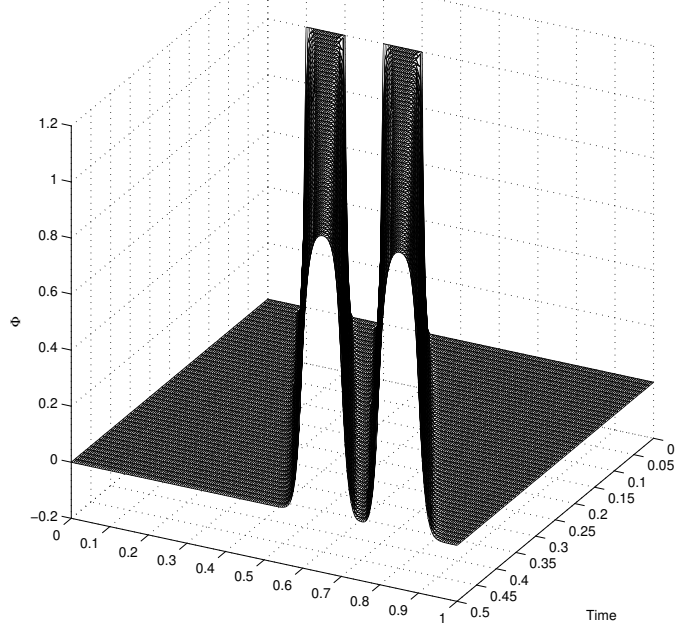

Figure 11: Transient 1D pure convective transport problem. The FIC-FEM solutions for a uniform mesh of 2002 -node elements and using the implicit midpoint rule $(\delta=0.5)$ are shown. Two different time steps, viz. $\Delta t=0.001 \mathrm{~s}$ and $\Delta t=0.0025 \mathrm{~s}$ were considered.

tial and parabolic layers. Exponential layers are typically found in the convection-dominant cases and near the boundary or close to regions where the source term is nonregular. Parabolic layers are found in the reaction-dominant cases near the boundary or close to the regions where the source term is nonregular and in convection-dominated cases along the characteristics of the solution. The characteristic internal boundary layers are typical of multidimensional problems. For this reason a direct extension of the stabilization procedure derived for 1D problems would not efficiently resolve these layers and the introduction of additional shock-capturing diffusion terms would be necessary in these cases $[14,40,69]$. This topic will be the subject of research of the authors in a subsequent work. 


\section{ACKNOWLEDGEMENTS}

The authors acknowledge the support for this research received from projects HFLUIDS of the Ministerio de Ciencia (Spain) and SAFECON, MODMAPS and COMP-DES-MAT of the European Research Council.

\section{References}

[1] Baiocchi C, Brezzi F, Franca LP. Virtual bubbles and the GaLS. Computer Methods in Applied Mechanics and Engineering 1993; 105:125-141.

[2] Boris JP, Book DL. Flux-corrected transport. I. SHASTA, A fluid transport algorithm that works. Journal of Computational Physics 1973; 11:38-69.

[3] Brezzi F, Bristeau MO, Franca LP, Mallet M, Rogé G. A relationship between stabilized finite element methods and the Galerkin method with bubble functions. Computer Methods in Applied Mechanics and Engineering 1992; 96:117-129.

[4] Brezzi F, Russo A. Choosing bubbles for advection-diffusion problems. Mathematical Models and Methods in Applied Sciences 1994; 4:571-587.

[5] Brezzi F, Hauke G, Marini LD, Sangalli G. Link-cutting bubbles for the stabilization of convection-diffusion-reaction problems. Mathematical Models and Methods in Applied Sciences $2003 ; \mathbf{1 3}(3): 445-461$.

[6] Brooks AN, Hughes TJR. Streamline Upwind/Petrov-Galerkin formulations for the convective dominated flows with particular emphasis on the incompressible Navier-Stokes equations. Computer Methods in Applied Mechanics and Engineering 1982; 32:199-259.

[7] Burman E, Ern A. Nonlinear diffusion and discrete maximum principle for stabilized Galerkin approximations of the convection-diffusion-reaction equation," Computer Methods in Applied Mechanics and Engineering 2002, 191, 3833-3855.

[8] Codina R. A discontinuity-capturing crosswind-dissipation for the finite element solution of the convection-diffusion equation," Computer Methods in Applied Mechanics and Engineering 1993, 110, 325-342.

[9] Codina R. Comparison of some finite element methods for solving the diffusion-convectionreaction equation, Computer Methods in Applied Mechanics and Engineering 1998, 156, 185210.

[10] Codina R. On stabilized finite element methods for linear systems of convection-diffusionreaction equations, Computer Methods in Applied Mechanics and Engineering 2000a, 190, 2681-2706.

[11] de Sampaio PAB, Coutinho ALGA. A natural derivation of discontinuity capturing operator for convection-diffusion problems, Computer Methods in Applied Mechanics and Engineering 2001, 190, 6291-6308.

[12] Doolan EP, Miller JJH, Schilders WHA. Uniform numerical methods for problems with initial and boundary layers. Dublin, Boole Press, 1980. Print.

[13] Donea J. A Taylor-Galerkin method for convective transport problems, International Journal for Numerical Methods in Engineering 1984, 20, 101-119.

[14] J. Donea, A. Huerta, Finite element method for flow problems. J. Wiley \& Sons, 2003. 
[15] Douglas J, Russell TF. Numerical methods for convection-dominated diffusion problems based on combining the method of characteristics with the finite element or finite difference procedures, SIAM Journal of Numerical Analysis 1982, 19, 871-885

[16] Dutra do Carmo EG, Alvarez GB. A new upwind function in stabilized finite element formulations, using linear and quadratic elements for the scalar convection-diffusion problems, Computer Methods in Applied Mechanics and Engineering 2004, 193, 2383-2402.

[17] Felippa C, Oñate E. Nodally exact Ritz discretizations of the 1D diffusion-absorption and Helmholtz equations by variational FIC and modified equation methods, Computational Mechanics 2007, 39, No.2, 91-111.

[18] Franca LP, Dutra do Carmo EG. The Galerkin gradient least-squares method, Computer Methods in Applied Mechanics and Engineering 1989, 74, 41-54.

[19] Franca LP, Farhat C. Bubble functions prompt unusual stabilized finite element methods, Computer Methods in Applied Mechanics and Engineering 1995, 123, 299-308.

[20] Franca LP, Valentin F. On an improved unusual stabilized finite element method for the advective-reactive-diffusive equation, Computer Methods in Applied Mechanics and Engineering 2001, 190, 1785-1800.

[21] Franz S, Hans-Görg R. The capriciousness of numerical methods for singular perturbations. SIAM Review (2011), 53(1):157-173.

[22] Galeão AC, Dutra do Carmo EG. A consistent approximate upwind Petrov-Galerkin method for the convection-dominated problems. Computer Methods in Applied Mechanics and Engineering 1988, 68, 83-95.

[23] Harari I, Hughes TJR. Stabilized finite element methods for steady advection-diffusion with production, Computer Methods in Applied Mechanics and Engineering 1994, 115, 165-191.

[24] Hauke G, García-Olivares A. Variational subgrid scale formulations for the advectiondiffusion-reaction equation, Computer Methods in Applied Mechanics and Engineering 2001, 190, 6847-6865.

[25] Hauke G. A simple subgrid scale stabilized method for the advection-diffusion-reaction equation, Computer Methods in Applied Mechanics and Engineering 2002, 191, 2925-2947.

[26] Hauke G, Sangalli G, Doweidar MH. Combining adjoint stabilized methods for the advectiondiffusion-reaction problem, Mathematical Models and Methods in Applied Sciences 2007, 17, No. 2, 305-326.

[27] Hughes TJR, Brooks AN. A theoretical framework for Petrov-Galerkin methods, with discontinuous weighting functions: application to the streamline upwind procedure, in Gallagher RH, Norrie DM, Oden JT, Zienkiewicz OC (eds.), Finite Elements in Fluids 1982, IV,Wiley, Chichester.

[28] Hughes TJR, Mallet M. A new finite element formulations for computational fluid dynamics: III. The generalized streamline operator for multidimensional advective-diffusive systems. Comput Methods Appl. Mech. Engrg. 1986a, 58, 305-328.

[29] Hughes TJR, Franca LP, Hulbert GM. A new finite element formulation for computational fluid dynamics: VIII. The Galerkin/least-squares method for advective-diffusive equations, Computer Methods in Applied Mechanics and Engineering 1989, 73, 173-189. 
[30] Hughes TJR, Feijoo GR, Mazzei L, Quincy JB. The variational multiscale method: a paradigm for computational mechanics, Computer Methods in Applied Mechanics and Engineering 1998, 166, 3-24.

[31] Idelsohn SR, Nigro N, Storti M, Buscaglia G. A Petrov-Galerkin formulation for advectivereaction-diffusion problems, Computer Methods in Applied Mechanics and Engineering 1996, $136,27-46$.

[32] Idelsohn SR, Heinrich JC, Oñate E. Petrov-Galerkin methods for the transient advectivediffusive equation with sharp gradients, International Journal for Numerical Methods in Engineering 1996, 39, 1455-1473.

[33] John V, Knobloch P. On spurious oscillations at layers diminishing (SOLD) methods for convection-diffusion equations: Part I - A review, Computer Methods in Applied Mechanics and Engineering 2007, 196, 2197-2215.

[34] Johnson C, Szepessy A, Hansbo P. On the convergence of shock-capturing streamline diffusion finite element methods for hyperbolic conservation laws, Mathematics of Computation 1990, $54,107-129$.

[35] Knobloch P. Improvements of the Mizukami-Hughes method for convection-diffusion equations, Computer Methods in Applied Mechanics and Engineering 2006, 196, 579-594.

[36] Labra C, Rojek J, Oñate E, Zárate F. Advances in discrete element modelling of underground excavations. Acta Geotechnica 2008: 3(4):317-322.

[37] Löhner R, Morgan K, Zienkiewicz OC. The solution of non-linear hyperbolic equation systems by the finite element method, International Journal for Numerical Methods in Fluids 1984, 4, 1043-1063.

[38] Mizukami A, Hughes TJR. A Petrov-Galerkin finite element method for convectiondominated flows: An accurate upwinding technique for satisfying the maximum principle, Computer Methods in Applied Mechanics and Engineering 1985, 50, 181-193.

[39] Nadukandi P, Oñate E, García Espinosa J. A high-resolution Petrov-Galerkin method for the $1 \mathrm{D}$ convection-diffusion-reaction problem, Computer Methods in Applied Mechanics and Engineering 2010, 199, 525-546.

[40] Nadukandi P, Oñate E, García Espinosa J. A high-resolution Petrov-Galerkin method for the convection-diffusion-reaction problem. Part II. A multidimensional extension, Computer Methods in Applied Mechanics and Engineering 2012, 213-216, 327-352.

[41] Oñate E, Idelsohn SR, Zienkiewicz OC, TaylorRL, Sacco C. A stabilized finite point method for analysis of fluid mechancis problems. Computer Methods in Applied Mechanics and Engineering 1996, 139, 315-346.

[42] Oñate E. Derivation of stabilized equations for numerical solution of advective-diffusive transport and fluid flow problems, Computer Methods in Applied Mechanics and Engineering 1998, 151, 233-265.

[43] Oñate E, Idelsohn SR. A mesh-free finite point method for advective-diffusive transport and fluid flow problems. Comput. Mech. 1998, 23, 283-292.

[44] Oñate E, Manzan M. A general procedure for deriving stabilized space-time finite element methods for advective-diffusive problems. Int. J. Num. Meth. Fluids 1999, 31, 203-221. 
[45] Oñate E, Manzan M. Stabilization techniques for finite element analysis of convectiondiffusion problems, in Sundén B, Comini G. (eds.), Computational Analysis of Convection Heat Transfer 2000, 71-117, WIT Press, Southampton (UK), 2000.

[46] Oñate E, Sacco C, Idelsohn SR. A finite point method for incompressible flow problems. Computing and Visualization in Science 2000, 2, 67-75.

[47] Oñate E, García J. A finite element method for fluid-structure interaction with surface waves using a finite calculus formulation. Computer Methods in Applied Mechanics and Engineering 2001, 191, 635-660.

[48] Oñate E, Perazzo F, Miquel J. A finite point method for elasticity problems. Computers and Structures 2001, 79, 2151-2163.

[49] Oñate E, Taylor RL, Zienkiewicz OC, Rojek J. A residual correction method based on finite calculus. Engineering Computations 2003, 20(5/6), 629-658.

[50] Oñate E. Possibilities of finite calculus in computational mechanics. International Journal for Numerical Methods in Engineering 2004, 60(1), 255-281.

[51] Oñate E, Idelsohn SR, Del Pin F, Aubry R. The particle finite element method. An overview. International Journal of Computational Methods 2004, 1(2), 267-307.

[52] Oñate E, Rojek J, Taylor RL Zienkiewicz OC. Finite calculus formulation for incompressible solids using linear triangles and tetrahedra. International Journal for Numerical Methods in Engineering 2004, 59, 1473-1500.

[53] Oñate E, Zarate F, Idelsohn SR. Finite element formulation for the convective-diffusive problems with sharp gradients using finite calculus, Computer Methods in Applied Mechanics and Engineering 2006, 195, 1793-1825.

[54] Oñate E, Miquel J, Hauke G. Stabilized formulation for the advection-diffusion-absorption equation using finite calculus and linear finite elements, Computer Methods in Applied Mechanics and Engineering 2006, 195, 3926-3946.

[55] Oñate E, Valls A, García J. FIC/FEM formulation with matrix stabilizing terms for incompressible flows at low and high Reynolds numbers. Computational Mechanics 2006, 38(4-5), 440-455.

[56] Oñate E, García J, Idelsohn SR, Del Pin F. FIC formulations for finite element analysis of incompressible flows. Eulerian, ALE and Lagrangian approaches. Computer Methods in Applied Mechanics and Engineering 2006, 195(23-24), 3001-3037.

[57] Oñate E, Miquel J, Zarate F. Stabilized solution of the multidimensional advection-diffusionabsorption equation using linear finite elements, Computers and Fluids 2007, 36, 92-112.

[58] Oñate E, Valls A, García J. Modeling incompressible flows at low and high Reynolds numbers via a finite calculus-finite element approach. Journal of Computational Physics 2007, 224, $332-351$.

[59] Oñate E, Idelsohn SR, Celigueta MA, Rossi R. Advances in the particle finite element method for the analysis of fluid-multibody interaction and bed erosion in free surface flows. Comput. Methods Appl. Mech. Engrg. 2008, 197(19-20), 1777-1800.

[60] Oñate E, Nadukandi P, Idelsohn S, García J, Felippa C. A family of residual-based stabilized finite element methods for Stokes flows. Int. J. Num. Meth. in Fluids 2011, 65 (1-3), 106-134. 
[61] Oñate E, Idelsohn SR, Felippa C. Consistent pressure Laplacian stabilization for incompressible continua via higher order finite calculus. International Journal for Numerical Methods in Engineering 2011, 87 (1-5), 171-195.

[62] Oñate E, Nadukandi P, Idelsohn S. P1/P0+ elements for incompressible flows with discontinuous material properties. Computer Methods in Applied Mechanics and Engineering 2014, 271, 185-209

[63] Shishkin GI, Grid approximation of singularly perturbed parabolic equations with internal layers. Russian Journal of Numerical Analysis and Mathematical Modelling 1988, 3(5):393408.

[64] Sun G, Stynes M. An almost fourth order uniformly convergent difference scheme for a semilinear singularly perturbed reaction-diffusion problem. Numerische Mathematik (1995), $70(4): 487-500$

[65] Tezduyar TE, Park YJ. Discontinuity-capturing finite element formulations for nonlinear convection-diffusion-reaction equations. Computer Methods in Applied Mechanics and Engineering 1986; 59:307-325.

[66] Yu CC, Heinrich JC. Petrov-Galerkin methods for the time-dependent convective transport equations, International Journal for Numerical Methods in Engineering 1986, 23, 883-901.

[67] Zienkiewicz OC, Codina R. A general algorithm for compressible and incompressible flows. Part I: the split, characteristic based scheme, International Journal for Numerical Methods in Fluids 1995, 20, 869-885.

[68] Zienkiewicz OC, Taylor RL, Zhu JZ. The finite element method. The basis. 6th Ed., Elsevier, 2005

[69] Zienkiewicz OC, Taylor RL, Nithiarasu P. The finite element method for fluid dynamics. 6th Ed., Elsevier, 2005.

\section{APPENDIX A}

\section{Appendix A Exact solution. Sourceless case $(Q=0)$}

Let us write the steady state form of the $1 \mathrm{D}$ convection-diffusion-reaction equation as

$$
k \phi^{\prime \prime}-u \phi^{\prime}-s \phi=0
$$

where a prime denotes differentiation with respect to the space coordinate $x$.

Eq.(A.1) can be written in non-dimensional form as

$$
\phi^{\prime \prime}-\frac{2 \gamma}{d} \phi^{\prime}-\frac{w}{d^{2}} \phi=0
$$

In Eq.(A.2), $d$ is an arbitrary distance such that $d=\Delta x=x_{i+1}-x_{i}=x_{i}-x_{i-1}$ in a grid of equally-spaced segments.

The non-dimensional parameter $\gamma$ and $w$ are therefore expressed as

$$
\gamma=\frac{u d}{2 k} \quad, \quad w=\frac{s d^{2}}{k}
$$

Note that $d=l^{e}$ for a mesh of 1D 2-noded linear elements.

The characteristic equations associated to the differential equation (A.2) is

$$
r^{2}-\frac{2 \gamma}{d} r-\frac{w}{d^{2}}=0
$$


From (A.4) we obtain

$$
r=\frac{1}{d}(\gamma \pm \lambda) \quad \text { with } \quad \lambda=\left(\gamma^{2}+w\right)^{1 / 2}
$$

The general solution of Eq.(A.2) is therefore

$$
\phi=A e^{\frac{x}{d}(\gamma+\lambda)}+B e^{-\frac{x}{d}(\gamma-\lambda)}
$$

where $A$ and $B$ are constants that depend on the boundary conditions of the problem.

For $x=x_{i}$ we have

$$
\hat{\phi}_{i}=\phi\left(x=x_{i}\right)=A e^{\frac{x_{i}}{d}(\gamma+\lambda)}+B e^{\frac{-x_{i}}{d}(\gamma-\lambda)}
$$

where $\hat{\phi}_{i}$ denotes the exact value of the transported variable at node $i$.

Similarly for $x=x_{i+1}=x_{i}+d$ and $x=x_{i-1}=x_{i}-d$ we obtain

$$
\begin{gathered}
\hat{\phi}_{i+1}=\phi\left(x=x_{i+1}\right)=A e^{\frac{x_{i}}{d}(\gamma+\lambda)} e^{(\gamma+\lambda)}+B e^{\frac{-x_{i}}{d}(\gamma-\lambda)} e^{(\gamma-\lambda)} \\
\hat{\phi}_{i-1}=\phi\left(x=x_{i-1}\right)=A e^{\frac{x_{i}}{d}(\gamma+\lambda)} e^{-(\gamma+\lambda)}+B e^{\frac{-x_{i}}{d}(\gamma-\lambda)} e^{-(\gamma-\lambda)}
\end{gathered}
$$

From Eqs.(A.7) and (A.8) we obtain

$$
\begin{aligned}
A e^{\frac{x_{i}}{d^{e}}(\gamma+\lambda)} & =-\frac{\hat{\phi}_{i} e^{(\gamma-\lambda)}-\hat{\phi}_{i+1}}{2 e^{\gamma} \sinh \lambda} \\
B e^{\frac{x_{i}}{d^{e}}(\gamma-\lambda)} & =-\frac{\hat{\phi}_{i+1}-\hat{\phi}_{i} e^{(\gamma+\lambda)}}{2 e^{\gamma} \sinh \lambda}
\end{aligned}
$$

Substituting Eqs.(A.10) and (A.11) into (A.9) gives

$$
\begin{aligned}
-\left(2 e^{\gamma} \sin \lambda\right) \hat{\phi}_{i-1} & =\hat{\phi}_{i}\left(e^{(\gamma-\lambda)} e^{-(\gamma+\lambda)}-e^{(\gamma+\lambda)} e^{-(\gamma-\lambda)}\right)+\hat{\phi}_{i+1}\left(e^{-(\gamma-\lambda)}-e^{-(\gamma+\lambda)}\right)= \\
& =\hat{\phi}_{i}\left(e^{-2 \lambda}-e^{2 \lambda}\right)+\hat{\phi}_{i+1} e^{-\gamma}\left(e^{\lambda}-e^{-\lambda}\right)=-2 \hat{\phi}_{i} \sinh (2 \lambda)+2 \hat{\phi}_{i+1} e^{-\lambda} \sinh \lambda
\end{aligned}
$$

Simplifying terms and taking into account that $\sinh (2 \lambda)=2 \sinh \lambda \cosh \lambda$ we finally obtain

$$
e^{\gamma} \hat{\phi}_{i-1}-(2 \cosh \lambda) \hat{\phi}_{i}+e^{-\gamma} \hat{\phi}_{i+1}=0
$$

which is the sought expression relating the exact numerical solution at three consecutive discrete points.

Expression (A.13) holds naturally for $\gamma^{2}+w \geq 0\left(\lambda\right.$ is a real number. For $\gamma^{2}+w<0(\lambda$ is a imaginary number), a similar expression is obtained simply changing $\cosh \lambda$ by $\cos |\lambda|$.

It is important to note that Eq.(A.13) holds for any value of the arbitrary distance $d$. In the paper we have taken $d=l^{e}$ for convenience, where $l^{e}$ is the element length in a uniform mesh of 2-noded elements. This allows us to interpret $\gamma$ as the element Peclet number and $w$ as the non-dimensional radiation parameter for the element (Eqs.(6)).

APPENDIX B Exact solution with source term $(Q \neq 0)$

$$
\begin{aligned}
\hat{\phi}_{i} & =A e^{(\gamma+\lambda) \frac{x_{i}}{d}}+B e^{(\gamma-\lambda) \frac{x_{i}}{d}}+\phi_{i}^{p} \\
\hat{\phi}_{i+1} & =A e^{(\gamma+\lambda) \frac{x_{i}}{d}} e^{(\gamma+\lambda)}+B e^{(\gamma-\lambda) \frac{x_{i}}{d}} e^{(\gamma-\lambda)}+\phi_{i+1}^{p} \\
\hat{\phi}_{i-1} & =A e^{(\gamma+\lambda) \frac{x_{i}}{d}} e^{-(\gamma+\lambda)}+B e^{(\gamma-\lambda) \frac{x_{i}}{d}} e^{-(\gamma-\lambda)}+\phi_{i-1}^{p}
\end{aligned}
$$


From (B.1) and (B.2)

$$
\begin{aligned}
A e^{(\gamma+\lambda) \frac{x_{i}}{d}} & =-\frac{\hat{\phi}_{i} e^{(\gamma-\lambda)}-\hat{\phi}_{i+1}-\phi_{i}^{p} e^{(\gamma-\lambda)}+\phi_{i+1}^{p}}{2 e^{\gamma} \sinh \lambda} \\
B e^{(\gamma-\lambda) \frac{x_{i}}{d}} & =-\frac{\hat{\phi}_{i+1}-\hat{\phi}_{i} e^{(\gamma+\lambda)}-\phi_{i+1}^{p}+\phi_{i}^{p} e^{(\gamma+\lambda)}}{2 e^{\gamma} \sinh \lambda}
\end{aligned}
$$

Substituting Eqs.(B.4) and (B.5) into (B.3) gives

$$
\begin{array}{r}
-\left(2 e^{\gamma} \sinh \lambda\right) \hat{\phi}_{i-1}=\left(\hat{\phi}_{i} e^{(\gamma-\lambda)}-\hat{\phi}_{i+1}-\phi_{i}^{p} e^{(\gamma-\lambda)}+\phi_{i+1}^{p}\right) e^{-(\gamma+\lambda)}+ \\
\left(\hat{\phi}_{i+1}-\hat{\phi}_{i} e^{(\gamma+\lambda)}-\phi_{i+1}^{p}+\phi_{i}^{p} e^{(\gamma+\lambda)}\right) e^{-(\gamma-\lambda)}-\phi_{i-1}^{p}\left(2 e^{\gamma} \sinh \lambda\right)
\end{array}
$$

After small algebra we obtain

$$
e^{\gamma} \hat{\phi}_{i-1}-(2 \cosh \lambda) \hat{\phi}_{i}+e^{-\gamma} \hat{\phi}_{i+1}=e^{\gamma} \phi_{i-1}^{p}-2 \cosh \lambda \phi_{i}^{p}+e^{-\gamma} \phi_{i+1}^{p}
$$

\section{APPENDIX C Stability conditions}

The characteristic equation associated to the stencil of Eq.(31) is

$$
-\left(\bar{\gamma}+1+\theta-\frac{w}{6}\right)-2\left(1+\theta+\frac{w}{3}\right) \rho+\left(-\bar{\gamma}+1+\theta-\frac{w}{6}\right) \rho^{2}=0
$$

with $\phi_{i}=\rho \phi_{i-1}$ and $\phi_{i+1}=\rho^{2} \phi_{i-1}$.

The general stability conditions are

$$
\begin{aligned}
\rho_{1} \rho_{2} & =\frac{\bar{\gamma}+1+\theta-\frac{w}{6}}{-\bar{\gamma}+1+\theta-\frac{w}{6}}>0 \\
\rho_{1}+\rho_{2} & =\frac{2\left(1+\theta+\frac{w}{3}\right)}{-\bar{\gamma}+1+\theta-\frac{w}{6}}>0
\end{aligned}
$$

where $\rho_{1}$ and $\rho_{2}$ are the roots of the characteristic equation (C.1).

Condition (C.2) is equivalent to

a.1)

$$
\bar{\gamma}+1+\theta-\frac{w}{6}>0 \rightarrow \theta>\frac{w}{6}-\bar{\gamma}-1
$$

and

$$
-\bar{\gamma}+1+\theta-\frac{w}{6}>0 \rightarrow \theta>\frac{w}{6}+\bar{\gamma}-1
$$

Hence

$$
\theta_{s}>\frac{w}{6}+|\bar{\gamma}|-1
$$

or

a.2)

$$
\bar{\gamma}+1+\theta-\frac{w}{6}<0 \quad \rightarrow \quad \theta<\frac{w}{6}-\bar{\gamma}-1
$$

and

$$
-\bar{\gamma}+1+\theta-\frac{w}{6}<0 \quad \rightarrow \quad \theta<\frac{w}{6}+\bar{\gamma}-1
$$

Hence

$$
\theta_{s}<\frac{w}{6}-\bar{\gamma}-1
$$

In Eqs.(C.6) and (C.9) $\theta_{s}$ is the stability value for $\theta$. 
On the other hand, condition (C.3) implies

b.1)

$$
1+\theta+\frac{w}{3}>0 \rightarrow \theta>-\frac{w}{3}-1
$$

and

$$
-\bar{\gamma}+1+\theta-\frac{w}{6}>0 \rightarrow \theta>\frac{w}{6}+\bar{\gamma}-1
$$

Hence

$$
\theta_{s}>\frac{w}{6}+\bar{\gamma}-1
$$

or

b.2)

and

$$
1+\theta+\frac{w}{3}<0 \rightarrow \theta<-\frac{w}{3}-1
$$

$$
-\bar{\gamma}+1+\theta-\frac{w}{6}<0 \rightarrow \theta<\frac{w}{6}+\bar{\gamma}-1
$$

Hence

$$
\theta_{s}<\frac{w}{3}-1
$$

Observation of Eqs.(C.6), (C.9), (C.12) and (C.15), and disregarding conditions (C.9) and (C.15), yields the general stability condition for all values of the velocity $u$, as

$$
\theta_{s}>\frac{w}{6}+|\bar{\gamma}|-1
$$

\section{APPENDIX D Computation of $\int_{l^{e}}|R| d x$}

In Eq.(83) we need to compute the integral $\int_{x_{1}}^{x_{2}}|R| d x$ where $|R|=\left|\frac{\hat{r}_{t}}{\hat{r}_{s}}\right|$ and $x_{i}($ with $i=1,2$ ) is the coordinate of node $i$ of an element.

Let us assume a linear variation for $R$ within the element as

$$
R(x)=a x+b
$$

We define

$$
\begin{aligned}
& R_{1}=R\left(x_{1}\right)=a x_{1}+b \\
& R_{2}=R\left(x_{2}\right)=a x_{2}+b
\end{aligned}
$$

From Eqs.(D.2) and (D.3) we deduce $a=\frac{R_{2}-R_{1}}{x_{2}-x_{1}}$.

We introduce the following change of variable

$$
R(x)=\xi=a x+b \quad, \quad d \xi=a d x
$$

Using Eq.(D.4) we find

$$
\int_{x_{1}}^{x_{2}}|R| d x=\frac{1}{a} \int_{\xi_{1}}^{\xi_{2}}|\xi| d \xi=\frac{1}{a} \int_{R_{1}}^{R_{2}}|\xi| d \xi
$$

The following conditions holds for a linear variation of a function $f$

$$
\int|f| d f=\frac{1}{2} f|f|
$$


Eq.(D.6) can be proved by noting that

$$
\frac{d}{d f}(f|f|)=2|f|
$$

Combining Eqs.(D.5), (D.6) and (D.7) gives

$$
\int_{x_{1}}^{x_{2}}|R| d x=\frac{l^{e}}{2}\left(\frac{R_{2}\left|R_{2}\right|-R_{1}\left|R_{1}\right|}{R_{2}-R_{1}}\right)
$$

where $l^{e}=x_{2}-x_{1}$.

It can also be proved that

$$
\lim _{R_{1} \rightarrow R_{2}} \frac{l^{e}}{2}\left(\frac{R_{2}\left|R_{2}\right|-R_{1}\left|R_{1}\right|}{R_{2}-R_{1}}\right)=l^{e}\left|R_{1}\right|=l^{e}\left|R_{2}\right|
$$

Eqs.(D.8) and (D.9) can be used for the analytical computation of the integral involving the discrete residuals in Eq.(83). 